FIAN/TD-06/14

ITEP/TH-15/14

\title{
Matching branches of non-perturbative conformal block at its singularity divisor
}

\author{
H.Itoyama, A.Mironov, A.Morozov" $\|$
}

August 29, 2021

\begin{abstract}
Conformal block is a function of many variables, usually represented as a formal series, with coefficients which are certain matrix elements in the chiral (e.g. Virasoro) algebra. Non-perturbative conformal block is a multi-valued function, defined globally over the space of dimensions, with many branches and, perhaps, additional free parameters, not seen at the perturbative level. We discuss additional complications of nonperturbative description, caused by the fact that all the best studied examples of conformal blocks lie at the singularity locus in the moduli space (at divisors of the coefficients or, simply, at zeroes of the Kac determinant). A typical example is the Ashkin-Teller point, where at least two naive non-perturbative expressions are provided by elliptic Dotsenko-Fateev integral and by the celebrated Zamolodchikov formula in terms of theta-constants, and they are different. The situation is somewhat similar at the Ising and other minimal model points.
\end{abstract}

\section{Introduction}

Conformal blocks are the central objects in $2 d$ conformal theories [1]: they are holomorphic constituents of the correlation functions, the latter being decomposed into bilinear combinations of the conformal blocks with different internal (intermediate) dimensions. Another ingredient of the theory are the structure constants, defining the coefficients in these expansions (for many purposes it is convenient not to include them into the normalization of conformal blocks, which is then chosen in some other way, more suitable from the point of view of complex analysis). The correlation function can be decomposed in several different ways, and the corresponding conformal blocks are related by linear transformations called modular transforms. Through the free fermion formalism [2], certain generating functions are interpreted as $\tau$-functions of the conventional integrable systems and the hierarchies of $\mathrm{KP} /$ Toda type, generalization of this formalism to the WZNW model $[3,4]$ should provide a description as non-Abelian $\tau$-functions of [5]. Long ago the conformal blocks were interpreted as states in the Hilbert space of $3 d$ Chern-Simons theory [6], thus providing an important ingredient of modern QFT approaches [7, 8, 9, 10, 11] to knot theory [12]. More recently, the AGT relations [13, 14, 15, $16,17,18,19]$ provided yet another interpretation of conformal blocks and their straightforward $q$-deformations $[20,21,22]$ as sums over instantons in respectively $4 d$ and $5 d$ Yang-Mills theories with extended supersymmetry $[23,24,25]$. The AGT correspondence proved to be useful in both direction: say, for using the sums over instantons for analysis of minimal models [15, 26, 27] and for using the Zamolodchikov solution [28] for analysis of instanton contributions in Seiberg-Witten theory in the conformal point [29, 30]. All these applications to quantum field theories in various dimensions explain the central role of conformal blocks in theoretical physics and the need for their thorough investigation. It was moved far enough, but, unfortunately, unfinished by Al.Zamolodchikov [31, 28, 32].

In this paper we concentrate on, perhaps, the simplest non-trivial of all conformal blocks: the 4-point spherical one, usually defined as a formal series in the double ratio $x=\frac{\left(x_{2}-x_{1}\right)\left(x_{3}-x_{4}\right)}{\left(x_{3}-x_{1}\right)\left(x_{2}-x_{4}\right)}$ of the four points on the Riemann sphere,

$$
B(x)=\sum_{k=0}^{\infty} B_{k} x^{k}
$$

\footnotetext{
$\S$ Department of Mathematics and Physics, Osaka City University and Osaka City University Advanced Mathematical Institute (OCAMI), Osaka, Japan; itoyama@sci.osaka-cu.ac.jp

『Lebedev Physics Institute and ITEP, Moscow, Russia; mironov@itep.ru; mironov@lpi.ru

"ITEP, Moscow, Russia; morozov@itep.ru
} 
The coefficients $B_{k}$ depend on the four external dimensions $\Delta_{1}, \ldots, \Delta_{4}$, on one internal dimension $\Delta$ and on the central charge $c$.

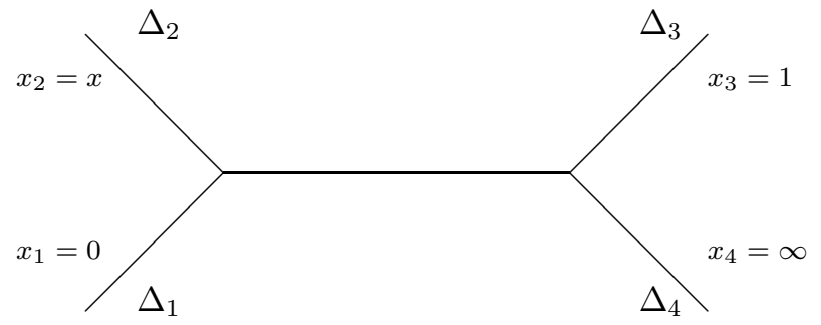

The series is not everywhere convergent, thus there is a question of what is the function $\mathcal{B}(x)$, which has $B(x)$ as its formal expansion near $x=0$. We call this function non-perturbative conformal block and give a short summary of its already known properties.

As a function of $x$, the 4-point spherical conformal block is believed to be analytic function ramified just at three points: $0,1, \infty$, with no essential singularities. Moreover, in the rational conformal models ramifications are of finite orders, thus such conformal blocks are actually the Belyi functions [33], appearing in consideration of Grothendieck's dessins d'enfant and the equilateral triangulations. The ramified coverings of $C P^{1}$, defined by the Belyi functions, are arithmetic curves, and their description in terms of conformal models and rational dimensions is a newly emerging interesting problem related to description of the universal moduli space [34, 35] and to modern theory of the Hurwitz numbers [36] and the Hurwitz $\tau$-functions [37, 38].

Coming back to non-perturbative $\mathcal{B}(x)$, the convergence of series (1.1) in $x$ is not uniform in the other parameters $\left(\Delta_{i}, \Delta, c\right)$, and this makes the entire function of all these variables quite sophisticated. $\mathcal{B}(x)$ is definitely non-trivial: it has many branches and changes under modular transformations. Moreover, this change can be represented as an integral transform in the internal dimension $\Delta$ :

$$
\begin{aligned}
\mathcal{B}_{\Delta}(1-x) & =\sum_{\Delta^{\prime}} \mathcal{M}_{\Delta}^{\Delta^{\prime}} \mathcal{B}_{\Delta^{\prime}}(x) \\
\mathcal{B}_{\Delta}\left(\frac{x}{x-1}\right) & =\sum_{\Delta^{\prime}} \mathcal{N}_{\Delta}^{\Delta^{\prime}} \mathcal{B}_{\Delta^{\prime}}(x)
\end{aligned}
$$

and in fact the non-perturbative modular kernels $\mathcal{M}_{\Delta}^{\Delta^{\prime}}$ and $\mathcal{N}_{\Delta}^{\Delta^{\prime}}$ are studied considerably better than $\mathcal{B}_{\Delta}(x)$ itself. The very fact that an $x$-independent modular kernel exists is highly non-trivial. This happens only if the intermediate (internal) dimensions $\Delta$ is chosen as a parameter in integral transformation (nothing like this would happen if we tried to use, say, one of $\Delta_{i}$ or $c$ ), and it reflects the associativity property of the operator product expansion of conformal field theory (it is also referred to as duality), or of the co-product in the Virasoro algebra, the modular kernel being the counterpart of the Racah coefficients (or $6 j$-symbols) in the theory of finite-dimensional Lie algebras.

In the context of quantum field theory and, in particular, in CFT one usually considers a given set of fields, i.e. fixes the set of external dimensions $\Delta_{i}$, while $\Delta$ remains arbitrary: it is common in this context to study the dependence on $\Delta$, but not so common to pay equal attention at dependencies on $\Delta_{i}$ or $c$, i.e. at the point in the "space of theories". Still, in modern stringy approaches it is more than natural just to discuss the $\mathcal{B}(\mu \mid x)$ on entire $6_{C} d$-space $\mathcal{M}$ of parameters $\mu=\left\{\Delta_{i}, \Delta, c\right\}$, without distinguishing the 1-dimensional "physical slice" $\mathcal{P} \subset \mathcal{M}$, where $\Delta_{i}, c=$ const. In fact, the conformal block should be analytically continued not only in $x$, but also in all these six extra parameters $\mu$. In particular, the modular kernel, which lives on the physical slice and thus already attracted certain attention, is believed to possess non-perturbative corrections as a function of $\Delta$ and $\Delta^{\prime}[39]$.

Thus, our goal in this paper is to attract attention at the non-perturbative conformal block $\mathcal{B}(\mu \mid x)$ not only on the physical slice $\mathcal{P} \subset \mathcal{M}$, but everywhere else, with the purpose of identifying the nature and essence of this important class of special functions. It turns out that even at the first step in this direction one runs into interesting details and this should stimulate more attention to such kind of problems.

There are special choices of external dimensions and central charge, when much seems to be known about $\mathcal{B}(x)$, and it is natural to begin from this point.

(A) For degenerate Verma modules there are null-vector constraints, which imply differential equations for the conformal block as functions of $x$ [1]. The order of the equation is defined by the level of the null-vector, and in these cases, it is, first, finite, and, second, greater than one. The second property means that $\mathcal{B}(x)$ has 
different branches and thus actually lives on a non-trivial Riemann surface in the $x$-space. The first property means that there is only a finite-dimensional family of solutions, while one could think that only one of the six parameters $\mu$ (that is, a particular external dimension, say, $\Delta_{2}$ ) is fixed by the null-vector condition, and a function of five remaining parameters should be a solution.

(B) The conformal block possesses the Dotsenko-Fateev representation in terms of multiple Selberg (generalized hypergeometric) integrals, analytically continued in the numbers $N_{1}, N_{2}$ of integration [17]. However, at $N_{1}$ and $N_{2}$ fixed they are just integrals and thus can be investigated as non-perturbative quantities by usual means of complex analysis. This is an obvious possibility, and it was not closely looked at because the "integral slice" $\mathcal{I}$ defined by $N_{1}, N_{2}=$ const is in a sense transversal to the physical slice $\mathcal{P}$, and one never looked carefully at the conformal blocks in transversal directions.

(C) Finally, there is the celebrated Zamolodchikov duality applicable to a very special (Ashkin-Teller) model with $c=1$ and $\Delta_{i}=\frac{1}{16}$. The point is that the operators of this dimension create square-root singularities for free fermions (there are two in the $c=1$ theory), and thus the correlator of fields $V_{1 / 16}$ at some points can be identified with the partition function of free fermions (Ising model) on a ramified covering of the original space-time. In the special case of 4-point correlator we get a torus with $x$-dependent modular parameter $\tau$, and in result the fantastic formula for the conformal block at Zamolodchikov's slice $\mathcal{Z} \subset \mathcal{M}$, with $\Delta_{i}=\frac{1}{16}, c=1$ :

$$
\mathcal{B}_{\Delta}(x)=\operatorname{Tr}_{\text {free fermions }} e^{i \pi \tau L_{0}}=\frac{q^{\Delta}}{\theta_{00}(q)}
$$

where

$$
q=e^{i \pi \tau}, \quad x=\frac{\theta_{10}^{4}(q)}{\theta_{00}^{4}(q)}
$$

and $\theta_{00}(q)=\sum_{n=-\infty}^{+\infty} q^{n^{2}}=\prod_{m=1}^{\infty}\left(1-q^{2 m}\right)\left(1+q^{2 m-1}\right)^{2}$. This is again a well-defined non-perturbative formula, and it is so famous because it is on the physical slice.

In this paper we discuss these examples and interplay between them. Our main observation is that both the minimal models (the typical example, where all external states are null-vectors) and Zamolodchikov's model at distinguished value of $\Delta=\frac{1}{4}$ appear to lie at the singular locus $\mathcal{L} \subset \mathcal{M}$ where coefficients of conformal blocks are ratios of two vanishing functions - thus they are ambiguously defined. This is a trivial, but previously underestimated phenomenon, because it is not seen at the physical slice: $\mathcal{L}$ and $\mathcal{P}$ are transversal when intersect. It is quite interesting to see what happens at these intersections. Moreover, it is plausible that future investigations of these singularities will help to understand the non-perturbative dependence of conformal blocks on $\mu$, which is important in order to shed some light on the somewhat mysterious non-perturbative expressions for the modular kernels (postulated by the identification [41, 40] of the Virasoro Racah matrices with those for peculiar representations of $S L(2))$.

In order to understand this phenomenon, one has to study the conformal block as a formal power series in $x$ in the vicinities of the singularities. They occur at the Kac zeroes, i.e. whenever the special relations between the internal dimension $\Delta$ and the central charge $c$ are satisfied. More concretely, if one parameterizes the conformal dimensions with the Dotsenko-Fateev parametrization:

$$
\Delta=\alpha\left(\alpha-b+\frac{1}{b}\right), \quad c=1-6\left(b-\frac{1}{b}\right)^{2}
$$

the Kac zeroes in $B_{k}$ happen at all integer $|m|>0,|n|>0$ such that $m n \leq k$ at the points

$$
\left.\alpha=\frac{1}{2}\left(\frac{n-1)}{b}-(m-1) b\right)\right)
$$

In order to have a non-singular answer, one has to require that the conformal block has additional zeroes at these singularities. Since the conformal block is a function of $x$, one has to require this for each singular $B_{k}$. In fact, as we shall see the Kac zeroes have an embedded structure: once $B_{k}$ is singular at some $k$, so are all $B_{i>k}$. Surprisingly enough, once one imposes the condition of vanishing the numerator of $B_{k}$ at the Kac zero, they also vanish for all $B_{i>k}$ ! Moreover, despite the answer depends on the direction of approaching the singularity, it is parameterized by just one arbitrary constant.

However, in the rational theories a new phenomenon occurs: there accidentally emerge some higher order poles (higher order Kac zeroes) due to the rational value of the central charge. Again, when this, say, double pole emerges at some $k$, it is also present at all $B_{i>k}$ until it becomes the third order pole etc. However, this is generically no longer the case for the corresponding zeroes in the numerator which are needed to cancel these 
poles: they are typically simple zeroes even in rational conformal theories, i.e. one meets in these cases the actual singularity. It means that in a consistent theory the corresponding structure constant should vanish. This makes the structure of the rational conformal theory in the vicinity of singularity quite sophisticated.

A notable exception is the minimal models. In the case of the minimal models the answer does not depend on the direction of approaching the singularity! This distinguishes these models and makes them unambiguously defined.

In the paper we illustrate the described picture with the concrete examples of the Ashkin-Teller model and the minimal models. Note that the Ashkin-Teller model is the only example manifestly known so far, when the answer is ambiguous near the singularity: the other known examples are the minimal models, when this ambiguity is absent.

\section{Series and "non-perturbative effects"}

We start with a general discussion of what is the non-perturbative conformal block, i.e. what is the conformal block outside its divergency radius ${ }^{1}$. The archetypical example of asymptotic perturbative series

$$
F(x)=\sum_{n=0}^{\infty} n ! x^{n}
$$

The factorial growth here is usually connected to the fact that the perturbation is performed with irrelevant operators, and a non-perturbative answer should have a different asymptotics at large values of fields.

The most obvious way to handle the series $(2.2)$ is to interpret the factorials as the $\Gamma$-function and use its integral representation to define the non-perturbative function:

$$
\mathcal{F}(x)=\sum_{n=0}^{\infty} \Gamma(n+1) x^{n}=\int_{0}^{\infty}\left(\sum_{n=0}^{\infty}(z x)^{n}\right) e^{-z} d z=\int_{0}^{\infty} \frac{e^{-z} d z}{1-z x}
$$

This answer, however, depends on the choice of the contour connecting 0 and $\infty$, and the ambiguity is just a residue at the singularity of the integrand:

$$
\mathcal{F}(x) \quad \text { is defined modulo } \quad \oint_{z=x^{-1}} \frac{e^{-z} d z}{1-z x} \sim \frac{e^{-1 / x}}{x}
$$

An alternative way to define the non-perturbative function $\mathcal{F}(x)$ is through writing an equation satisfied by the perturbative series. In the case of $(2.2)$ the simplest one is the first order differential equation:

$$
x \frac{d}{d x} x F(x)=\sum_{n=0}^{\infty}(n+1) ! x^{n+1}=F(x)-1
$$

Solutions to this equation depend on one free constant: the freedom is to add (with an arbitrary coefficient) a solution to the homogeneous equation

$$
x^{2} \frac{d F_{0}}{d x}=(1-x) F_{0}(x) \quad \Longrightarrow \quad F_{0}(x)=\frac{e^{-1 / x}}{x}
$$

i.e. $\mathcal{F}(x)$ is once again defined modulo $x^{-1} e^{-1 / x}$. The slight difference is that in the first approach the coefficient seems to be arbitrary integer, while in the second approach, i.e. when the non-perturbative function is defined as a $D$-module, as a solution to some linear equation, it can naturally be arbitrary complex number.

Of course, in both cases we considered a minimal definition: the non-perturbative function can be lifted to bigger moduli spaces, for example, by considering equations of higher order, which have more solutions. Still,

\footnotetext{
${ }^{1}$ Note here that the divergency radius of the conformal block depends on the chosen variable. For instance, in variable $x$ this radius does not exceed 1 , since there is a singularity at $x=1$. At the same time, if one uses the variable $q=e^{\pi i \tau}$ related to $x$ by the formula

$$
\begin{gathered}
x=\frac{\theta_{10}^{4}}{\theta_{00}^{4}} \\
\theta_{10}(q)=\theta_{10}(0 \mid \tau)=2 q^{1 / 4} \sum_{n=0} q^{n(n+1)}=2 q^{1 / 4} \prod_{m=1}^{\infty}\left(1-q^{2 m}\right)\left(1+q^{2 m}\right)^{2}
\end{gathered}
$$

the series converge in the entire region where $\mathcal{B}(x)$ is analytic, as was demonstrated by Al.Zamolodchkov [28].
} 
usually there is no a natural way to get rid of extra parameters; or, whenever this is done, there should be some additional reason for such a restriction. Thus, the moral at this stage is:

the non - perturbative function usually depends on extra hidden parameters

We emphasize that this fact is implicit in any non-perturbative consideration. It is enough to remind the celebrated example of instantons in Yang-Mills theories: not only they come with the typical non-perturbative weight $e^{-1 / g^{2}}$, they bring in an additional parameter, not seen at the perturbative level: the $\theta$-angle. In fact, restriction to real angles (i.e. to unimodular free coefficients in front of the instanton contribution) is somewhat similar to restriction to integer-valued coefficients in the above example, i.e. is motivated more by the concrete method of analytical continuation than by the essence of the problem. What is important in this example, it emphasizes the physical relevance of additional (perturbatively hidden) parameters: they affect the nonperturbative renormalization [42], whose significance is nowadays well appreciated and widely investigated with the help of Seiberg-Witten theory [43]-[45].

In application to conformal blocks, this would imply that $\mathcal{B}(\mu \mid x)$ is actually $\mathcal{B}(\mu|x| C)$, i.e. the nonperturbative conformal block depends on additional parameters $C$ not seen at perturbative level, and not present in the original expansion (1.1) derived from representation theory of the Virasoro algebra.

However, one should expect that the conformal theory is essentially free $[46,47,48,4]$, therefore there is no room for a factorial growth of expansions in the coupling constants of the irrelevant operators. Thus, nonperturbative hidden parameters do not appear in this context, and all ambiguities are of a different nature. As we shall see, this makes the non-perturbative conformal blocks easier comprehensible than non-perturbative effects in more general interacting models of quantum field theory. As already mentioned, the expansion series (1.1) in $x$ are believed to have finite radia of convergence, and in this sense they are somewhat different from (2.2).

In fact, even for (2.2) it is recently shown [38]) that it can be naturally associated with a certain $\mathrm{KP} /$ Toda $\tau$-function (arising in a simplified counting problem of the Belyi functions [49]). Since the $\tau$-functions satisfy quadratic Hirota relations [50, 2, 5] they are not preserved by linear transformations, and this can provide a new interesting tool to restrict non-perturbative ambiguities (perhaps, even to distinguish the choice of $\theta=0$ in instanton calculus, thus leading to a new kind of $\theta$-problem solution).

\section{Perturbative expansion of conformal block}

We begin from reminding the basic facts about the perturbative conformal block (1.1).

\subsection{Definition of perturbative block for four primaries}

The basic definition of the 4-point conformal block comes from the bilinear expansion of the correlation function of four primary fields [1]

$$
\begin{gathered}
\left\langle V_{\Delta_{1}, \bar{\Delta}_{1}}\left(x_{1}, \bar{x}_{1}\right) V_{\Delta_{2}, \bar{\Delta}_{2}}\left(x_{2}, \bar{x}_{2}\right) V_{\Delta_{3}, \bar{\Delta}_{3}}\left(x_{3}, \bar{x}_{3}\right) V_{\Delta_{4}, \bar{\Delta}_{4}}\left(x_{4}, \bar{x}_{4}\right)\right\rangle= \\
=\sum_{\Delta, \bar{\Delta}} C_{12}^{\Delta, \bar{\Delta}} C_{34}^{\Delta, \bar{\Delta}} \times G_{\Delta}\left(\Delta_{1}, \Delta_{2}, \Delta_{3}, \Delta_{4} ; c ; x_{1}, x_{2}, x_{3}, x_{4}\right) \times \bar{G}_{\bar{\Delta}}\left(\bar{\Delta}_{1}, \bar{\Delta}_{2}, \bar{\Delta}_{3}, \bar{\Delta}_{4} ; c ; \bar{x}_{1}, \bar{x}_{2}, \bar{x}_{3}, \bar{x}_{4}\right)
\end{gathered}
$$

where $C_{12(34)}^{\Delta, \bar{\Delta}}$ are the structure constants of the theory which are sometimes included in the definition of the conformal block. However, in principle, the structure constants is a separate object, it defines the concrete conformal theory, and it is a separate problem to list all admissible structure constants (they should satisfy additional complicated restrictions like duality). At the same time, the conformal block $G_{\Delta}\left(\Delta_{1}, \Delta_{2}, \Delta_{3}, \Delta_{4} ; c ; x_{1}, x_{2}, x_{3}, x_{4}\right)$ is a universal function of four points $x_{i}$ that depends on 6 parameters: 5 dimensions and the central charge, it encodes only properties of the Virasoro algebra. In fact, it is a non-trivial function of the double-ratio $x=\frac{\left(x_{2}-x_{1}\right)\left(x_{3}-x_{4}\right)}{\left(x_{3}-x_{1}\right)\left(x_{2}-x_{4}\right)}$ only:

$$
G_{\Delta}\left(\Delta_{1}, \Delta_{2}, \Delta_{3}, \Delta_{4} ; c ; x_{1}, x_{2}, x_{3}, x_{4}\right)=\left(\prod_{i<j} x_{i j}^{\zeta_{i j}}\right) B_{\Delta}(x)=\left(\prod_{i<j} x_{i j}^{\zeta_{i j}}\right) \sum_{k} x^{k} B_{k}\left(\Delta_{1}, \ldots, \Delta_{4} \mid \Delta, c\right)
$$

Here $x_{i j} \equiv x_{i}-x_{j}, \zeta_{12(13)}=0, \zeta_{14}=-2 \Delta_{1}, \zeta_{23}=\Delta_{4} \Delta_{1}-\Delta_{2}-\Delta_{3}, \zeta_{24}=\Delta_{1}+\Delta_{3}-\Delta_{2}-\Delta_{4}, \zeta_{34}=$ $\Delta_{1}+\Delta_{2}-\Delta_{3}-\Delta_{4}$. Note that permutations of points gives rise to the modular transformations (1.2). For 
instance, upon permuting $x_{1}$ and $x_{3}$ we obtain

$$
B\left(\Delta_{3}, \Delta_{2}, \Delta_{1}, \Delta_{4} \mid \Delta, c ; 1-x\right)=\sum_{\Delta^{\prime}} \mathcal{M}_{\Delta}^{\Delta^{\prime}} B\left(\Delta_{1}, \Delta_{2}, \Delta_{3}, \Delta_{4} \mid \Delta^{\prime}, c ; x\right)
$$

From now on we omit the parameters $\Delta_{1}, \Delta_{2}, \Delta_{3}, \Delta_{4}, c$ in notation of the conformal block unless it may lead to a misunderstanding.

The conformal block expansion (3.1) is derived by using the operator product expansion (OPE)

$$
\begin{gathered}
V_{\Delta_{1} \Delta_{2}}\left(x_{1}, \bar{x}_{1}\right) V_{\Delta_{2}, \bar{\Delta}_{2}}\left(x_{2}, \bar{x}_{2}\right)=\sum_{\Delta} C_{12}^{\Delta, \bar{\Delta}}\left(x_{1}-x_{2}\right)^{\Delta-\Delta_{1}-\Delta_{2}}\left(\bar{x}_{1}-\bar{x}_{2}\right)^{\bar{\Delta}-\bar{\Delta}_{1}-\bar{\Delta}_{2} \times} \\
\times\left(\sum_{Y, \bar{Y}}\left(x_{1}-x_{2}\right)^{|Y|}\left(\bar{x}_{1}-\bar{x}_{2}\right)^{|\bar{Y}|} \beta_{Y}\left(\Delta \mid \Delta_{1}, \Delta_{2}\right) \bar{\beta}_{\bar{Y}}\left(\bar{\Delta} \mid \bar{\Delta}_{1}, \bar{\Delta}_{2}\right) \hat{L}_{-Y} \hat{\bar{L}}_{-\bar{Y}} V_{\Delta_{2}, \bar{\Delta}_{2}}\left(x_{2}, \bar{x}_{2}\right)\right)
\end{gathered}
$$

where the bracket at the r.h.s. is a sum over descendants, which are labeled by Young diagrams $(Y, \bar{Y})$ of all sizes $\left(|Y|,|\bar{Y}|\right.$. For simplicity we consider only the OPE of the primary fields $V_{1}$ and $V_{2}$ : this is possible if we restrict ourselves to the 4-point conformal blocks only, which we really do in this paper.

With the OPE, one can project the products $V_{1}\left(x_{1}\right) V_{2}\left(x_{2}\right)$ and $V_{3}\left(x_{3}\right) V_{4}\left(x_{4}\right)$ onto Verma modules given by $V_{\Delta, \bar{\Delta}}$ and $V_{\Delta^{\prime}, \bar{\Delta}^{\prime}}$ correspondingly, and since the pair correlation function of any fields is non-zero iff the both fields have the same conformal dimension, one finally obtains (3.1), and the conformal block represents a chiral part of the correlation function with specified intermediate dimension. It can be defined completely within the chiral algebra [51].

In terms of the chiral algebra one defines the conformal block through a chiral correlator:

$$
B_{\Delta}(x)=\left\langle\left. V_{\Delta_{1}}\left(x_{1}\right) V_{\Delta_{2}}\left(x_{2}\right)\right|_{\Delta} V_{\Delta}\left(x_{3}\right) V_{\Delta\left(x_{4}\right)}\right\rangle
$$

where hereafter we denote the corresponding chiral primary fields with the same letter $V$, and the chiral OPE looks like

$$
V_{\Delta_{1}}\left(x_{1}\right) V_{\Delta_{2}}\left(x_{2}\right) \stackrel{\Delta}{\longrightarrow}\left(x_{1}-x_{2}\right)^{\Delta-\Delta_{1}-\Delta_{2}}\left(\sum_{Y}\left(x_{1}-x_{2}\right)^{|Y|} \beta_{Y}\left(\Delta \mid \Delta_{1}, \Delta_{2}\right) \hat{L}_{-Y} V_{\Delta}\left(x_{1}\right)\right)
$$

The coefficients $\beta_{Y}$ are easily related with the three point functions: one suffices to consider the three point function $\Gamma_{Y}\left(\Delta_{1}, \Delta_{2} \mid \Delta\right)=\left\langle V_{\Delta_{1}} V_{\Delta_{2}} \mid \hat{L}_{-Y} V_{\Delta}\right\rangle$ and use (3.6). Then, one immediately obtains for the three point function

$$
\Gamma_{Y}\left(\Delta_{1}, \Delta_{2} \mid \Delta\right)=\sum_{Y^{\prime}} T_{Y, Y^{\prime}}(\Delta) \beta_{Y^{\prime}}\left(\Delta \mid \Delta_{1}, \Delta_{2}\right)
$$

with the Shapovalov matrix

$$
T_{Y, Y^{\prime}}(\Delta)=\left\langle\hat{L}_{-Y} V_{\Delta} \mid \hat{L}_{-Y^{\prime}} V_{\Delta}\right\rangle
$$

Note that picking up a single term in the sum over $\Delta$ may lead to losing associativity of the product, which in simplest examples is guaranteed by the structure constants $C_{12}^{\Delta, \bar{\Delta}}$. For many models (when there are degenerate representations of the Virasoro algebra in the spectrum) they vanish for most values of $\Delta, \bar{\Delta}$. However, in these cases it is often enough to just impose selection rules on $\Delta$. In any case, for the 4-point conformal blocks one can avoid using associativity. At this stage one gets

$$
B_{\Delta}\left(\Delta_{i} \mid x\right)=\sum_{Y, Y^{\prime}} x_{12}^{|Y|} x_{34}^{\left|Y^{\prime}\right|} \beta_{Y}\left(\Delta \mid \Delta_{1}, \Delta_{2}\right) \beta_{Y^{\prime}}\left(\Delta \mid \Delta_{3}, \Delta_{4}\right)\left\langle\hat{L}_{-Y} V_{\Delta} \mid \hat{L}_{-Y^{\prime}} V_{\Delta}\right\rangle=\sum_{Y, Y^{\prime}} \Gamma_{Y}\left(\Delta_{1}, \Delta_{2} \mid \Delta\right) T_{Y, Y^{\prime}}^{-1} \Gamma_{Y^{\prime}}\left(\Delta_{3}, \Delta_{4} \mid \Delta\right)
$$

At last, in order to calculate the 3-point functions, one needs to use properties of the chiral algebra:

- consistency of the Virasoro algebra with the scalar product $<\ldots>$ :

$$
\left\langle\hat{L}_{Y} V \mid V^{\prime}\right\rangle=\left\langle V \mid \hat{L}_{-Y} V^{\prime}\right\rangle
$$

for arbitrary operators $V$ and $V^{\prime}$ (not necessarily primary). We normalize the primaries so that $\left\langle V_{\Delta} \mid V_{\Delta}\right\rangle=1$. In fact, just this identity implies that the scalar product in (3.8) is equal to the Shapovalov matrix

$$
\left\langle\hat{L}_{-Y} V_{\Delta} \mid \hat{L}_{-Y^{\prime}} V_{\Delta}\right\rangle=\left\langle V_{\Delta} \mid \hat{L}_{Y} \hat{L}_{-Y^{\prime}} V_{\Delta}\right\rangle,
$$


in particular it vanishes for Young diagrams of differing sizes $|Y| \neq|\bar{Y}|$, what is important for the projective invariance of the correlator, i.e. for collecting the four coordinates $x_{1}, \ldots, x_{4}$ into a single double ratio $x=\frac{\left(x_{2}-x_{1}\right)\left(x_{3}-x_{4}\right)}{\left(x_{3}-x_{1}\right)\left(x_{2}-x_{4}\right)}$, which allows one to put $x_{1}=0, x_{2}=x, x_{3}=1, x_{4}=\infty$.

- The comultiplication for the Virasoro algebra [51], which is a direct consequence of the Ward identities [1]:

$$
\hat{L}_{n}\left(V_{1}(0) V_{2}(x)\right)=\left(\sum_{k=0}^{\infty} x^{n+1-k}\left(\begin{array}{c}
n+1 \\
k
\end{array}\right) \hat{L}_{k-1} V_{1}(0)\right) V_{2}(x)+V_{1}(0) \hat{L}_{n} V_{2}(x)
$$

Applying these postulates, one immediately obtains $[1,52,53]$ that for $Y=\left\{y_{1} \geq y_{2} \geq \ldots\right\}$

$$
\Gamma_{Y}\left(\Delta_{1}, \Delta_{2} \mid \Delta\right)=\prod_{i}\left(\Delta+y_{i} \Delta_{2}-\Delta_{1}-\sum_{j<i} y_{j}\right)
$$

\subsection{Chain-vectors}

Alternatively, one can use a very effective representation of $B_{k}$ in (1.1) via scalar products of constituents of the distinguished chain-vectors [1]

$$
B_{k}=\left\langle k, \Delta_{3}, \Delta_{4} \mid k, \Delta_{1}, \Delta_{2}\right\rangle
$$

which depend also on $\Delta$ and $c$ and satisfy simple defining recurrence relations

$$
\begin{aligned}
\hat{L}_{n}\left|k, \Delta_{1}, \Delta_{2}\right\rangle= & \left(k-n+\Delta+n \Delta_{2}-\Delta_{1}\right)\left|k-n, \Delta_{1}, \Delta_{2}\right\rangle, \quad 0<n \leq k, \\
& \hat{L}_{0}\left|k, \Delta_{1}, \Delta_{2}\right\rangle=(k+\Delta)\left|k, \Delta_{1}, \Delta_{2}\right\rangle
\end{aligned}
$$

The possibility for such recurrence relations to unambiguously define a chain of such states within Verma module is a prominent feature of Virasoro algebra, which holds also for $\widehat{U(1)}$ but not for higher $W_{N}$ algebras, beginning from $W_{3}$. In fact, the chain vector is basically nothing but the projection of the operator product expansion of two primaries with conformal dimensions $\Delta_{1}, \Delta_{2}$ onto a third one with dimension $\Delta$ :

$$
\begin{gathered}
\hat{V}_{\Delta_{1}}(0) \hat{V}_{\Delta_{2}}(x) \stackrel{\Delta}{\longrightarrow} x^{\Delta-\Delta_{1}-\Delta_{2}} \sum_{Y} x^{|Y|} \beta_{Y}\left(\Delta \mid \Delta_{1}, \Delta_{2}\right) \hat{L}_{-Y} \hat{V}_{\Delta}(0), \\
\left|k, \Delta_{1}, \Delta_{2}\right\rangle=\sum_{|Y|=k} \beta_{Y}\left(\Delta \mid \Delta_{1}, \Delta_{2}\right) \hat{L}_{-Y} \hat{V}_{\Delta}(0)
\end{gathered}
$$

i.e.

$$
B_{k}=\sum_{\left|Y_{1}\right|=\left|Y_{2}\right|=k} \beta_{Y}\left(\Delta \mid \Delta_{1}, \Delta_{2}\right) \beta_{Y}\left(\Delta \mid \Delta_{3}, \Delta_{4}\right)\left\langle\hat{V}_{\Delta} \hat{L}_{Y_{1}} \mid \hat{L}_{-Y_{2}} \hat{V}_{\Delta}(0)\right\rangle
$$

Importance of the chain vectors becomes especially clear within the AGT conjecture, since projectors of the chain vectors for product of the Virasoro and Heisenberg algebras on peculiar states in the Verma module associated with the generalized Jack polynomials reproduce the Nekrasov functions,

$$
N_{Y_{1}, Y_{2}}=\left\langle k, \Delta_{3}, \Delta_{4} \mid J_{Y_{1}, Y_{2}}\right\rangle\left\langle J_{Y_{1}, Y_{2}} \mid k, \Delta_{1}, \Delta_{2}\right\rangle
$$

(here pairs of Young diagrams emerge due to the product of the Virasoro and Heisenberg algebras) and they have extra poles not present in the scalar products (3.14). For various realizations of this idea see [19]. ${ }^{2}$

\footnotetext{
${ }^{2}$ For instance, at the level one:

$$
\left|J_{0,1}\right\rangle=\left(\hat{L}_{-1}+(Q+2 a) \hat{a}_{-1}\right)|a\rangle \quad\left|J_{1,0}\right\rangle=\left(\hat{L}_{-1}+(Q-2 a) \hat{a}_{-1}\right)|a\rangle
$$

i.e.

$$
\hat{L}_{-1}|a\rangle=-\frac{1}{4 a}\left((Q-2 a)\left|J_{0,1}\right\rangle-(Q+2 a)\left|J_{1,0}\right\rangle\right) \quad \hat{a}_{-1}|a\rangle=\frac{1}{4 a}\left(\left|J_{0,1}\right\rangle-\left|J_{1,0}\right\rangle\right)
$$
}




\subsection{Explicit expressions}

In result we get for the first several coefficients $B_{k}$ in

$$
B(x)=1+\sum_{k=1}^{\infty} B_{k} x^{k}
$$

the following explicit expressions:

$$
\begin{gathered}
B_{1}=\frac{\left(\Delta+\Delta_{2}-\Delta_{1}\right)\left(\Delta+\Delta_{3}-\Delta_{4}\right)}{2 \Delta} \\
\mathcal{B}_{\Delta}^{(2)}=\frac{\left(\Delta+\Delta_{2}-\Delta_{1}\right)\left(\Delta+\Delta_{2}-\Delta_{1}+1\right)\left(\Delta+\Delta_{3}-\Delta_{4}\right)\left(\Delta+\Delta_{3}-\Delta_{4}+1\right)}{4 \Delta(2 \Delta+1)}+ \\
+\frac{\left[\left(\Delta_{1}+\Delta_{2}\right)(2 \Delta+1)+\Delta(\Delta-1)-3\left(\Delta_{2}-\Delta_{1}\right)^{2}\right]\left[\left(\Delta_{3}+\Delta_{4}\right)(2 \Delta+1)+\Delta(\Delta-1)-3\left(\Delta_{3}-\Delta_{4}\right)^{2}\right]}{2(2 \Delta+1)(2 \Delta(8 \Delta-5)+(2 \Delta+1) c)}
\end{gathered}
$$

Denominators of these expressions,

$$
\begin{gathered}
K_{2}=4 \Delta\left(16 \Delta^{2}-10 \Delta+2 c \Delta+c\right), \\
K_{3}=6\left(3 \Delta^{2}+c \Delta-7 \Delta+c+2\right) \cdot K_{2}, \\
K_{4}=4(8 \Delta+c-1)\left(16 \Delta^{2}-82 \Delta+10 c \Delta+15 c+66\right) \cdot K_{3},
\end{gathered}
$$

at generic values of $c$ possess only simple zeroes, which coincide with zeroes of the Kac determinants $K D_{n}$ (i.e. determinants of Shapovalov matrices), though these latter sometimes have non-zero multiplicities. These extra (multiplicity of) zeroes of the Kac determinants cancel against zeroes of the numerators in expressions for the 4-point conformal blocks, e.g.

$$
\begin{gathered}
K D_{3}=2 \Delta \cdot K_{3}, \\
K D_{4}=4 \Delta^{2}\left(16 \Delta^{2}-10 \Delta+2 c \Delta+c\right) \cdot K_{4}
\end{gathered}
$$

Since the cancelation takes place at arbitrary points of the moduli space $\mathcal{M}$ (i.e. for arbitrary dimensions and central charges), these zeroes play no role in further our considerations, and we sometimes call the reduced quantities (3.24) the Kac determinants assuming that this should not cause any confusion.

\subsection{Coefficients $B_{k}$ from Dotsenko-Fateev representation of [17]}

Let us make the change of variables

$$
\Delta_{\mu}=\alpha_{\mu}\left(\alpha_{\mu}-Q\right), c=1-6 Q^{2}, Q=b-\frac{1}{b}
$$

with $\alpha_{i}$ constrained by

$$
\begin{gathered}
\alpha-\alpha_{1}-\alpha_{2}=b N_{1}, \\
Q-\alpha-\alpha_{3}-\alpha_{4}=b N_{2}
\end{gathered}
$$

In fact, one can choose in (3.27) $Q-\alpha_{i}$ instead of any $\alpha_{i}$, since there is a symmetry in the theory w.r.t. this operation (in particular, (3.26) remains unchanged under this transformation).

With this change of variables (3.26) $B_{k}\left(\Delta_{1}, \ldots \Delta_{4}, \Delta, c\right)$ turns into rational functions of $J_{k}\left(\alpha_{1}, \alpha_{2}+\right.$ $\left.\alpha_{3}, b, N_{1}, N_{2}\right)$,

$$
B_{k}=J_{k}
$$


which at integer non-negative values of $N_{1}$ and $N_{2}$ coincide with the values of Selberg-Kadell[54] integrals, $N_{1}$ times between 0 and $x$ and $N_{2}$ times between 0 and 1 . This fact $[17,55]$ can be interpreted as Dotsenko-Fateev like representation of conformal blocks [46] via conformal matrix model of $[56,16]$.

In more detail,

$$
\begin{gathered}
J_{k}=\frac{Z(v)}{Z(0)}, \\
Z(v)=\int \prod_{a<a^{\prime}}\left(v_{a}-v_{a^{\prime}}\right)^{2 b^{2}} \prod_{a} v_{a}^{2 \alpha_{1} b}\left(x-v_{a}\right)^{2 \alpha_{2} b}\left(1-v_{a}\right)^{2 \alpha_{3} b} d v_{a}
\end{gathered}
$$

and $N_{1}$ integrations here runs 0 to $x$, while $N_{2}$ integrations goes from 0 to 1 . Note that the integrals are not obligatory around the closed contours: for irrational products $\alpha_{i} \alpha_{j}$ these are not so easy to define. In other words, following [17] we define the integrals in the same way as the archetypical $B$-function integral

$$
\int_{0}^{1} z^{a-1}(1-z)^{b-1} d z=\frac{\Gamma(a) \Gamma(b)}{\Gamma(a+b)}
$$

is defined. The new point is analytical continuation in $N_{1}$ and $N_{2}$ : since $B_{k}$ are rational functions of these variables, continuation is straightforward and unambiguous.

These formulas can also be straightforwardly $q$-deformed $[21,22]$, this generalization is related to $5 d$ gauge theories, to $q$-Virasoro algebras, to MacDonald polynomials and DAHA.

\subsection{General structure of $B_{k}$}

The poles of $B_{k}$ (Kac zeroes) have a very simple form in the Dotsenko-Fateev parametrization: they occur at all integers $|m|>0,|n|>0$ such that $m n \leq k$ at the points

$$
\alpha_{m, n}=\frac{1}{2}\left(\frac{n-1}{b}-(m-1) b\right)
$$

It follows that once a pole appears at some $B_{k}$, it also happens at all $B_{l>k}$. Such a singularity in the conformal block at level $k$ can be removed by a proper choice of the external dimensions $\Delta_{i}$ so that the theory would still make sense. Surprisingly enough, once the pole disappears in this way at the level $k$, it simultaneously disappears at all higher levels. In other words, not only the Kac zeroes, but also the zeroes of the numerators have a nested structure. In the next sections we study this phenomenon is detail, in particular, we observe that in conformal theories with rational values of the central charge there emerge multiple Kac zeroes, which sometimes still cancel out with multiple zeroes of the numerator, but sometimes not.

Our goal in the next sections is to look for a non-perturbative parameters in the conformal block, i.e. we look for conformal blocks which are not uniquely defined functions of $x$ at given $\Delta_{i}, \Delta, c$. One immediate example is provided in the point $\Delta_{i}=1 / 16, \Delta=1 / 4, c=1$ (see s.4): this is the case of Ashkin-Teller model solved by Al.Zamolodchikov [32]. On the other hand, at the same point there is another conformal block, given by an elliptic integral which follows from formulas in [17]. The existence of two different solutions may imply that this would provide us with needed non-perturbative parameter. However, as we explain later in s.5, this is not the case: the ambiguity is just due to different possibilities of approaching the singularity, since the Ashkin-Teller model is located in the moduli space exactly at the Kac zero.

Another possibility to look for non-perturbative parameters could be in the cases when some of the external dimensions correspond to a degenerate vector. If this vector is degenerate at level $k$ and one specially matches the intermediate dimension, there is a differential equation of order $k$ in $x$ for the conformal block. This equations has generally $k$ independent solutions and, hence, the full answer would be a linear combination of these, depending on $k-1$ arbitrary constants. However, these constants can not be associated with nonperturbative ambiguity in the definition of the conformal block, since each of these $k$ solutions correspond to exactly one conformal block fixed by a proper asymptotics! Moreover, the modular transformation $x \rightarrow 1-x$ transforms any of these conformal blocks through remaining $k-1$.

In fact, the absence of non-perturbative parameter in the second case is not surprising: we already mentioned that as a function of $x$ the 4-point spherical conformal block is believed to be a function, ramified just at three points: $0,1, \infty$, with no essential singularities. Hence, the expansion series in $x$ have finite radia of convergence, and in this sense they are somewhat different, say, from (2.2). However, the convergence in $x$ is not uniform in the other parameters $\left(\Delta_{i}, \Delta, c\right)$, and this makes the entire function of all variables quite sophisticated, which we observe in the Ashkin-Teller case. 


\section{Non-perturbative parameters. First quest: Ashkin-Teller model}

\subsection{Elliptic integrals}

Note that (3.29) is an integral representation of entire conformal block $\mathcal{B}(x)$, not of individual coefficients $J_{k}=B_{k}$ of its $x$-expansion. A natural idea could be to use such representation as an obvious candidate for a non-perturbative definition.

Of course, this idea raises a number of interesting questions - especially, about the analytical continuation in $N$ and associated non-perturbative dependencies on intermediate dimensions, like $\Delta$. This would open a way to study non-perturbative modular kernels $[40,41,57,59,39]$ (which perturbatively are just Fourier transforms $[58])$.

However, before going deeper in that direction, it makes sense to look at this approach in a less controversial situation: at natural (positive integer) values of $N_{1}$ and $N_{2}$. It deserves beginning from the simplest case of $c=1$, i.e. $b=1$. In this case there is a solution of (3.27) at $N_{1}=1, N_{2}=0$.

Even after that there is a further simplification: for a special choice of external dimensions, when the integral becomes elliptic.

For instance, at $\alpha_{i}=-1 / 4$ the integral (3.29) turns into just an ordinary elliptic integral

$$
K(x)=\int \frac{d z}{y(z)}
$$

with $y^{2}(z)=z(1-z)(z-x)$, which is elementary to analyze, both perturbatively and non-perturbatively.

$$
\int_{0} \frac{d z}{y(z)}=\sum c_{k}^{2} x^{k}=1+\frac{1}{4} x+\frac{9}{64} x^{2}+\ldots
$$

and

$$
\begin{gathered}
\mathcal{B}^{\text {ell }}(x)=(1-x)^{1 / 8} K(x)=1+\frac{1}{8} x+\frac{7}{128} x^{2}+\frac{33}{1024} x^{3}+\frac{713}{32768} x^{4}+\frac{4165}{262144} x^{5}+ \\
+\frac{51205}{4194304} x^{6}+\frac{326255}{33554432} x^{7}+\frac{17078585}{2147483648} x^{8}+\frac{114071265}{17179869184} x^{9}+\ldots
\end{gathered}
$$

The MAPLE command to generate this formula is

series $\left((1-\mathrm{x})^{\wedge}(1 / 8) * \operatorname{EllipticK}(\operatorname{sqrt}(\mathrm{x})) / \mathrm{Pi} * 2, \mathrm{x}, 10\right)$;

For non-perturbative analysis of integrals the best method is via the Picard-Fuchs equations. The PicardFuchs equation for

$$
\Pi=\oint_{C} \frac{d z}{y(z)}=\oint_{C} \frac{d z}{\sqrt{z(1-z)(z-x)}}
$$

along any closed contour $C$ is

$$
\left(x(1-x) \frac{\partial^{2}}{\partial x^{2}}+(1-2 x) \frac{\partial}{\partial x}+\frac{1}{4}\right) \Pi=0
$$

There are two solutions: one is $K(x)$ having the asymptotics 1 at small $x$, the other one, $K^{\prime}(x)$ has the asymptotics $\log x[60]$. We are definitely interested in the first case.

\subsection{Zamolodchikov's formula}

In [28] Al.Zamolodchikov suggested a wonderful formula for the non-perturbative conformal block at the slice where $\Delta_{i}=\frac{1}{16}, \quad c=1$ :

$$
\mathcal{B}_{\Delta}^{Z a m}(x)=\mathcal{B}_{\Delta}\left(\Delta_{i}=\frac{1}{16}, c=1 \mid x\right)=(1-x)^{-1 / 8} \frac{(16 q / x)^{\Delta}}{\theta_{00}(q)}
$$

where relation between $x$, considered as a ramification point, and elliptic parameter $q=e^{i \pi \tau}$ is given by

$$
x=\frac{\theta_{10}^{4}}{\theta_{00}^{4}}=16 q \cdot \frac{\left(1+q^{2}+q^{6}+\ldots\right)^{4}}{\left(1+2 q+2 q^{4}+\ldots\right)^{4}}=16 q-128 q^{2}+\ldots
$$


and the theta-constants

$$
\begin{gathered}
\theta_{00}(q)=\theta_{00}(0 \mid \tau)=1+2 \sum_{n=1}^{\infty} q^{n^{2}}=\prod_{m=1}^{\infty}\left(1-q^{2 m}\right)\left(1+q^{2 m-1}\right)^{2} \\
\theta_{01}(q)=\theta_{01}(0 \mid \tau)=1+2 \sum_{n=1}^{\infty}(-)^{n} q^{n^{2}}=\prod_{m=1}^{\infty}\left(1-q^{2 m}\right)\left(1-q^{2 m-1}\right)^{2} \\
\theta_{10}(q)=\theta_{10}(0 \mid \tau)=2 q^{1 / 4} \sum_{n=0} q^{n(n+1)}=2 q^{1 / 4} \prod_{m=1}^{\infty}\left(1-q^{2 m}\right)\left(1+q^{2 m}\right)^{2} \\
\theta_{11}^{\prime}(q)=\theta_{00} \theta_{01} \theta_{10}=2 q^{1 / 4} \prod_{m=1}^{\infty}\left(1-q^{2 m}\right)^{3}=\eta^{3}(q)
\end{gathered}
$$

Equation (4.7) relates the modular transformations of $x$, generated by $x \longrightarrow 1-x$ and $x \longrightarrow-1 / x+1$ to the modular transformations of theta constants generated by $\theta_{\epsilon, \delta}(0 \mid-1 / \tau)=(-i)^{\epsilon \delta} \sqrt{-i \tau} \theta_{\delta, \epsilon}(0 \mid \tau)$ and $\theta_{\epsilon, \delta}(0 \mid \tau+1)=e^{\pi i / 4 \epsilon} \theta_{\epsilon, \delta+1-\epsilon}(0 \mid \tau)$, where the characteristics of the $\theta$-functions $\epsilon$ and $\delta$ are understood as taken by modulo 2 . The crucial role in this relation is played by the Riemann identity $\theta_{00}^{4}(0 \mid \tau)=\theta_{01}^{4}(0 \mid \tau)+\theta_{10}^{4}(0 \mid \tau)$.

At the Ashkin-Teller point $\mu_{A T}$, where additionally $\Delta=\frac{1}{4}$,

$$
(1-x)^{1 / 8} \mathcal{B}_{1 / 4}^{Z a m}=\frac{(16 q / x)^{1 / 4}}{\theta_{00}}=\frac{2 q^{1 / 4}}{\theta_{10}}=\frac{1}{1+q^{2}+q^{6}+\ldots}
$$

and perturbative expansion near in powers of $x$ is

$$
\begin{aligned}
B_{1 / 4}^{\text {Zam }}(x) & =\left(1+\frac{1}{8} x+\frac{9}{128} x^{2}+\ldots\right)\left(1-\frac{1}{256} x^{2}+\ldots\right)=1+\frac{1}{8} x+\frac{17}{256} x^{2}+\frac{93}{2048} x^{3}+\frac{2269}{65536} x^{4}+ \\
& +\frac{14705}{524288} x^{5}+\frac{198109}{8388608} x^{6}+\frac{1370655}{67108864} x^{7}+\frac{77366631}{4294967296} x^{8}+\frac{554104463}{34359738368} x^{9}+\ldots
\end{aligned}
$$

The MAPLE command to generate this formula is

$\mathrm{q}:=\mathrm{x}->$ EllipticNome $(\mathrm{x})$;

$A:=x->(1-x)^{\wedge}(-1 / 8) *(16 * q(\operatorname{sqrt}(x)) / x)^{\wedge}(1 / 4) / \operatorname{JacobiTheta3}(0, q(\operatorname{sqrt}(x)))$;

$\operatorname{series}(A(x), x, 10)$;

\subsection{Intersection of physical and elliptic slices}

\subsubsection{The problem}

Now we can compare the two expressions (4.3) and (4.10). Both correspond to the Ashkin-Teller point

$$
\mu_{A T}: \quad \Delta_{i}=\frac{1}{16}, \quad \Delta=\frac{1}{4}, \quad c=1
$$

in the moduli space $\mathcal{M}$, both are expansions of the well defined and well known functions of $x$, but they are different already in the second terms of the $x$-expansion!

What does this mean? How could we get two different expressions for $B_{2}$ at the given point?

The reason is that $\mu_{A T}$ actually lies on the divisor of $B_{2}$. In order to obtain the second coefficient of the $x$-expansion from (3.23) one needs to resolve the singularity $\frac{0}{0}$, and the resolution is ambiguous. Of course, this is a usual situation for a function of many variables (even rational, like $B_{k}$ ), still this causes additional ambiguities in the conformal block: it is not uniquely defined by the conformal dimensions at the Kac zeroes.

Our immediate goal in this situation is to study the vicinity of $\mu_{A T}$ and see how the two expressions (4.3) and (4.10) emerge from a single (3.23). At the next step we should look at other coefficients $B_{k}$ and at other interesting points on the divisor.

\subsection{2 $\quad B_{2}$ in the vicinity of $\mu_{A T}$}

Thus, we substitute

$$
\Delta_{i}=\frac{1}{16}+\epsilon \delta_{i}, \quad \Delta=\frac{1}{4}+\epsilon \delta, \quad c=1+\epsilon \sigma
$$


into expression $(3.22),(3.23), \ldots$ for the coefficients of the $x$-expansion of conformal block and look at what happens at small values of $\epsilon$.

For $B_{1}$ we get nothing interesting:

$$
B_{1}^{A T}=\frac{1}{8}+O(\epsilon)
$$

and this is exactly what is needed in both (4.3) and (4.10)

However, for $B_{2}$ the situation gets far more interesting:

$$
B_{2}^{A T}=\frac{\frac{25}{256} \sigma \epsilon+O\left(\epsilon^{2}\right)}{\frac{3}{2} \sigma \epsilon+O\left(\epsilon^{2}\right)} \stackrel{?}{=} \frac{25}{384}+O(\epsilon)
$$

This is still another rational number different from those in both (4.3) and (4.10).

However, let us still look at the next order in $\epsilon$ :

$$
B_{2}^{A T}=\frac{\frac{25}{256} \sigma \epsilon+\rho \epsilon^{2}+O\left(\epsilon^{3}\right)}{\frac{3}{2} \sigma \epsilon+\left(16 \delta^{2}+8 \sigma \delta\right) \epsilon^{2}+O\left(\epsilon^{3}\right)}
$$

where

$$
\rho=\frac{1}{16}\left(17 \delta^{2}-2 \delta\left(\delta_{1}+\delta_{2}+\delta_{3}+\delta_{4}\right)+12\left(\delta_{1}+\delta_{2}\right)\left(\delta_{3}+\delta_{4}\right)\right)+\frac{15}{32} \sigma\left(2 \delta-\delta_{1}+\delta_{2}+\delta_{3}-\delta_{4}\right)
$$
Then

Now one can see what happens. In both cases (4.3) and (4.10) one approach the point $\mu_{A T}$ keeping $\sigma=0$.

$$
B_{2}^{A T}(c=1)=\frac{1}{256}\left(17-2 \frac{\delta_{1}+\delta_{2}+\delta_{3}+\delta_{4}}{\delta}+12 \frac{\left(\delta_{1}+\delta_{2}\right)\left(\delta_{3}+\delta_{4}\right)}{\delta^{2}}\right)+O(\epsilon)
$$

Zamolodchikov's formula (4.10) refers to the physical slice, when the theory and observables (external dimensions $\Delta_{i}$ ) are fixed: thus, $\sigma=0$ and $\delta_{1}=\ldots=\delta_{4}=0$, and only the intermediate dimension could vary. In other words, only $\delta$ is imagined to be non-vanishing. When we approach $\mu_{A T}$ from this special direction we get

$$
\lim _{\delta \rightarrow 0} B_{2}^{A T}\left(\Delta_{i}=\frac{1}{16}, c=1\right)=\frac{17}{256}
$$

i.e. the answer is (4.10).

For the elliptic integral (4.3) the situation is absolutely different. What is fixed in this case is the number of integrations, $N_{1}=1, N_{2}=0$. According to (3.27) this implies that we approach $\mu_{A T}$ from a very different direction, where

$$
\begin{gathered}
\sigma=0, \\
\delta+2\left(\delta_{1}+\delta_{2}\right)=0, \\
\delta-2\left(\delta_{3}+\delta_{4}\right)=0
\end{gathered}
$$

Note that (3.27) is written in terms of $\alpha$-parameters, not dimensions, and at $c=1$ our $\delta_{i}=\frac{\alpha_{i}-\alpha_{i}^{A T}}{2 \epsilon \sqrt{\Delta_{i}}}$, hence, the additional coefficient $2=\frac{\sqrt{1 / 4}}{\sqrt{1 / 16}}$ in (4.19). Then (4.17) gives

$$
\lim _{\delta \rightarrow 0} B_{2}^{A T}((4.19), c=1)=\frac{1}{256}\left(17-\frac{12}{2^{2}}\right)=\frac{7}{128}
$$

i.e. exactly what is needed for (4.3). Note that (4.19) imposes only two constraints, but this turns to be enough to provide an unambiguous limit in (4.17).

In fact, it is both convenient and natural to put the central charge and the dimensions on equal footing. If we parameterize $c=1-6(b-1 / b)^{2}$, see (3.26), and use $b=1+\epsilon \eta$ instead of $c=1+\epsilon \sigma$, deviations from the AT point will be entirely of the order $\epsilon^{2}$ in the numerator and denominator. In other words,

$$
\sigma=-24 \eta^{2} \epsilon
$$

and this is the resolution of singularity that we use in the rest of this section. In particular, in this parametrization

$$
B_{2}^{A T}=\frac{\frac{17}{16} \delta^{2}-\frac{75}{32} \eta^{2}-\frac{1}{8} \delta\left(\delta_{1}+\delta_{2}+\delta_{3}+\delta_{4}\right)+\frac{3}{4}\left(\delta_{1}+\delta_{2}\right)\left(\delta_{3}+\delta_{4}\right)+O(\epsilon)}{16 \delta^{2}-36 \eta^{2}+O(\epsilon)}
$$

where we omitted the common overall factors $\epsilon^{2}$ in the numerator and denominator. 


\subsubsection{Other $B_{k}$ : universality and the germ of conformal block at $\mu_{A T}$}

If one now makes the same substitution in $B_{3}$, one again obtains the double zeroes at $\mu_{A T}$ in the numerator and denominator, and

$$
B_{3}^{A T}=\frac{\frac{7533}{1024} \delta^{2}-\frac{32805}{2048} \eta^{2}-\frac{729}{512} \delta\left(\delta_{1}+\delta_{2}+\delta_{3}+\delta_{4}\right)+\frac{2187}{256}\left(\delta_{1}+\delta_{2}\right)\left(\delta_{3}+\delta_{4}\right)+O(\epsilon)}{162 \delta^{2}-\frac{729}{2} \eta^{2}+O(\epsilon)}
$$

The numbers can look ugly, however they are in fact just the same as in (4.22):

$$
B_{3}^{A T}=-\frac{15}{512}+\frac{9}{8} \cdot B_{2}^{A T}+O(\epsilon)
$$

This means that we do not need to perform any independent calculation in the third terms in (4.3) and (4.10): inter-relation between these two cases is fully fixed at the level of the second coefficient.

Indeed, for $B_{4}$ the same property persists for higher $B_{k}$ :

$$
\begin{gathered}
B_{1}=\frac{1}{8}, \quad B_{2}=\frac{17-r}{256}, \quad B_{3}=\frac{93-9 r}{2^{12}}, \quad B_{4}=\frac{2269-281 r}{2^{16}}, \\
B_{5}=\frac{14705-2125 r}{2^{19}}=\frac{5 \cdot 17}{2^{19}} \cdot(173-25 r), \quad \ldots
\end{gathered}
$$

where

$$
r \equiv \frac{-3 \eta^{2}+8 \delta\left(\delta_{1}+\delta_{2}+\delta_{3}+\delta_{4}\right)-48\left(\delta_{1}+\delta_{2}\right)\left(\delta_{3}+\delta_{4}\right)}{4 \delta^{2}-9 \eta^{2}}
$$

It is natural to assume that this remains true in general:

$$
B_{k}=B_{k}^{Z a m}+\frac{r}{3}\left(B_{k}^{e l l}-B_{k}^{Z a m}\right), \quad k<6
$$

However, this is actually true only for $k<6$. Indeed, at level 6 there Kac zero at $\Delta=1 / 4$ becomes of the fourth order: $(n-m)^{2} / 4=1 / 4$ when $n=2, m=1$, i.e. at level $n \cdot m=2$; when $n=3, m=2$, i.e. at level $n \cdot m=6$ etc. The numerator still cancels this multiple zero at the Ashkin-Teller point, however, the ambiguity becomes a ratio of two quartic polynomials of $\delta_{i}, \delta, \eta$. For $6 \leq k<12$ we have:

$$
B_{k}=B_{k}^{Z a m}+\frac{r}{3}\left(B_{k}^{e l l}-B_{k}^{Z a m}\right)+\frac{r_{2}}{3 \cdot 2^{11}} C_{k}, \quad 6 \leq k<12
$$

with

$$
C_{k<6}=0, \quad C_{6}=1, \quad C_{7}=\frac{25}{8}, \quad C_{8}=\frac{1577}{800} C_{7}, \ldots
$$

where

$$
r_{2}=\frac{P_{4}\left(\eta, \delta, \delta_{i}\right)}{\left(4 \delta^{2}-9 \eta^{2}\right)\left(4 \delta^{2}-25 \eta^{2}\right)}
$$

and

$$
\begin{gathered}
P_{4}\left(\eta, \delta, \delta_{i}\right)=-60 \eta^{4}+\eta^{2}\left(15 \delta^{2}+86 \delta\left(\delta_{1}+\delta_{2}+\delta_{3}+\delta_{4}\right)+120\left(\delta_{1}+\delta_{2}\right)^{2}+120\left(\delta_{3}+\delta_{4}\right)^{2}-660\left(\delta_{1}+\delta_{2}\right)\left(\delta_{3}+\delta_{4}\right)\right)- \\
-8 \delta^{3}\left(\delta_{1}+\delta_{2}+\delta_{3}+\delta_{4}\right)+48 \delta^{2}\left(3\left(\delta_{1}+\delta_{2}\right)\left(\delta_{3}+\delta_{4}\right)-\left(\delta_{1}+\delta_{2}\right)^{2}-\left(\delta_{3}+\delta_{4}\right)^{2}\right)- \\
-192 \delta\left(\delta_{1}+\delta_{2}\right)\left(\delta_{3}+\delta_{4}\right)\left(\delta_{1}+\delta_{2}+\delta_{3}+\delta_{4}\right)+960\left(\delta_{1}+\delta_{2}\right)^{2}\left(\delta_{3}+\delta_{4}\right)^{2}
\end{gathered}
$$

The moral of this story, is that the behavior of conformal block in the vicinity of the point $\mu_{A T}$ is universal: does not depend on the order $k$ of the $x$-expansion. This opens a possibility to suggest a formula for the germ of conformal block at $\mu_{A T}$ (the next $r_{3}$ emerge at level 12, when the Kac zero gets multiplicity 6 ):

$$
\begin{gathered}
B(x)=B^{\text {Zam }}(x)+\frac{r}{3}\left(B^{\text {ell }}(x)-B^{\text {Zam }}(x)\right)+ \\
+\frac{r_{2}}{3} \cdot \frac{x^{6}}{2^{11}}\left(1+\frac{25}{8} x+\frac{1577}{256} x^{2}+\frac{20141}{2048} x^{3}+\frac{911193}{65536} x^{4}+\frac{9549597}{524288} x^{5}+\ldots\right)+x^{12} B_{12}+\ldots+O(\epsilon)
\end{gathered}
$$


Zamolodchikov's expansion corresponds to all external $\delta_{i}=0$, while the elliptic locus in the vicinity of the AT point is a union of several hyperplanes, each defined by two conditions:

$$
\sum_{i=1}^{4} \delta_{i}=-\frac{3}{2} \eta, \quad \delta=2\left(\delta_{3}+\delta_{4}\right)+\eta
$$

or

$$
\sum_{i=1}^{4} \delta_{i}=\frac{3}{2} \eta, \quad \delta+2\left(\delta_{3}+\delta_{4}\right)=2 \eta
$$

\subsection{Chain vectors}

As an alternative to the above technique, one can perform an analysis of the singularity locus in terms of the representation of $B_{k}$ in (1.1) via the chain-vectors (s.3.2). For instance, at the Ashkin-Teller point the leading behaviour of the chain-vectors is given by

$$
\begin{gathered}
\beta_{2}=-\frac{\zeta}{\xi}+O(\xi) \\
\beta_{11}=\frac{\zeta}{\xi}+O(\xi) \\
\beta_{21}=-\frac{\zeta}{2 \xi}+O(\xi) \\
\beta_{111}=\frac{\zeta}{2 \xi}+O(\xi) \\
\beta_{3}=0
\end{gathered}
$$

where

$$
\zeta=\frac{\delta-6 \delta_{1}-6 \delta_{2}}{2\left(32 \delta^{2}+3 \eta\right)}
$$

and $\xi$ is a distance from the singularity locus. Thus, the chain-vectors are non-zero vectors nearby the locus, but they must have zero norm in the leading order, since their norm (which is equal to the conformal block (3.14)) is finite on the locus. Indeed, the singularities cancel in the conformal block, because of degeneracy of the Shapovalov matrix (since the singularity locus is located in zeroes of determinant of the Shapovalov matrix)

$$
\left(\sum_{|Y|=k} \operatorname{sing}\left(\beta_{Y}\right)\right)^{2}=0
$$

\section{Perturbative conformal block in the vicinity of Kac divisor}

\subsection{Poles of $B_{k}$ and their nested structure}

We already discussed in s.3 and demonstrated in the manifest Ashkin-Teller example in s.4 that

- if a zero appears in the Kac determinant $K_{k}$, it persists in all higher $K_{k}$ with $k \geq m$. The Kac determinants depend only on the intermediate dimension $\Delta$ and the central charge $c$, and in the $\alpha$-parametrization (3.26) the zeroes are actually at the points (3.30). At such points all the coefficients $B_{k}$ with $k \geq m$ are singular.

- However, one can adjust external dimensions $\Delta_{i}$ so that the numerator in $B_{m}$ also vanishes. What happens is that then it also vanishes in the numerators of all higher $B_{k}$ with $k \geq m$.

For example, a Kac zero at the third level is at

$$
\alpha_{1,3}=-b
$$

The numerator of $B_{3}$ at this zero is

$$
X=\frac{X_{12} X_{34}}{24 b^{5}\left(b^{2}-1\right)\left(3 b^{2}-1\right)\left(4 b^{4}-1\right)}
$$


with

$$
X_{12}=\left(\alpha_{1}-\alpha_{2}\right)\left(b^{2}-1-b \alpha_{1}-b \alpha_{2}\right)\left(2 b^{2}-1-b \alpha_{1}-b \alpha_{2}\right)\left(b+\alpha_{1}-\alpha_{2}\right)\left(b-\alpha_{1}+\alpha_{2}\right)\left(1+b \alpha_{1}+b \alpha_{2}\right)
$$

The numerator of $B_{4}$ is the same $X$, multiplied by

$$
\frac{\left(2 b^{3}+2 b+\alpha_{2}^{2} b-\alpha_{2} b^{2}+\alpha_{2}-\alpha_{1}^{2} b+\alpha_{1} b^{2}-\alpha_{1}\right)\left(2 b^{3}+2 b+\alpha_{3}^{2} b-\alpha_{3} b^{2}+\alpha_{3}-\alpha_{4}^{2} b+\alpha_{4} b^{2}-\alpha_{4}\right)}{4 b^{2}\left(b^{2}+1\right)}
$$

This demonstrates that at the Kac zero of level $m=3$ the zero of the numerator of $B_{m}$ (the zero of $X$ ) remains a zero of the higher $B_{k}$, e.g. of $B_{4}$.

Once again, not only the zero loci $V\left(K_{k}\right)$ of Kac determinants are nested,

$$
V\left(K_{k}\right) \subset V\left(K_{l}\right) \quad \text { or } \quad K_{l} \vdots K_{k} \quad \text { for } \quad l>k
$$

the same is true for the numerators of $B_{k}$, provided they are restricted to $V\left(K_{k}\right)$ : for $\mathcal{V}_{k}=\left\{\right.$ zeroes of $\left.\left.B_{k}\right|_{V\left(K_{k}\right)}\right\}$ we have

$$
\mathcal{V}_{k} \subset \mathcal{V}_{l} \quad \text { for } \quad k<l
$$

\subsection{Coefficients $B_{k}$ at intersection of zeroes of numerators and denominators}

At generic point of $\bigcup_{k} V\left(K_{k}\right)$ the coefficients of conformal block are singular (have poles), thus this union form a singularity locus in the moduli space $\mathcal{M}$ and it has codimension one. However, there is a codimension one hypersurface within the singularity locus (thus it has codimension two in $\mathcal{M}$ ), where the numerators are also vanishing. And at these points we have an ambiguity of the type $0 / 0$, the value of the coefficients depending on the direction from which one approaches such point in $\mathcal{M}$. It is natural to name this codimension-two hypersurface the ambiguity locus. Our next goal is to describe behavior of the entire conformal block, not just of its particular coefficients $B_{k}$, at this locus.

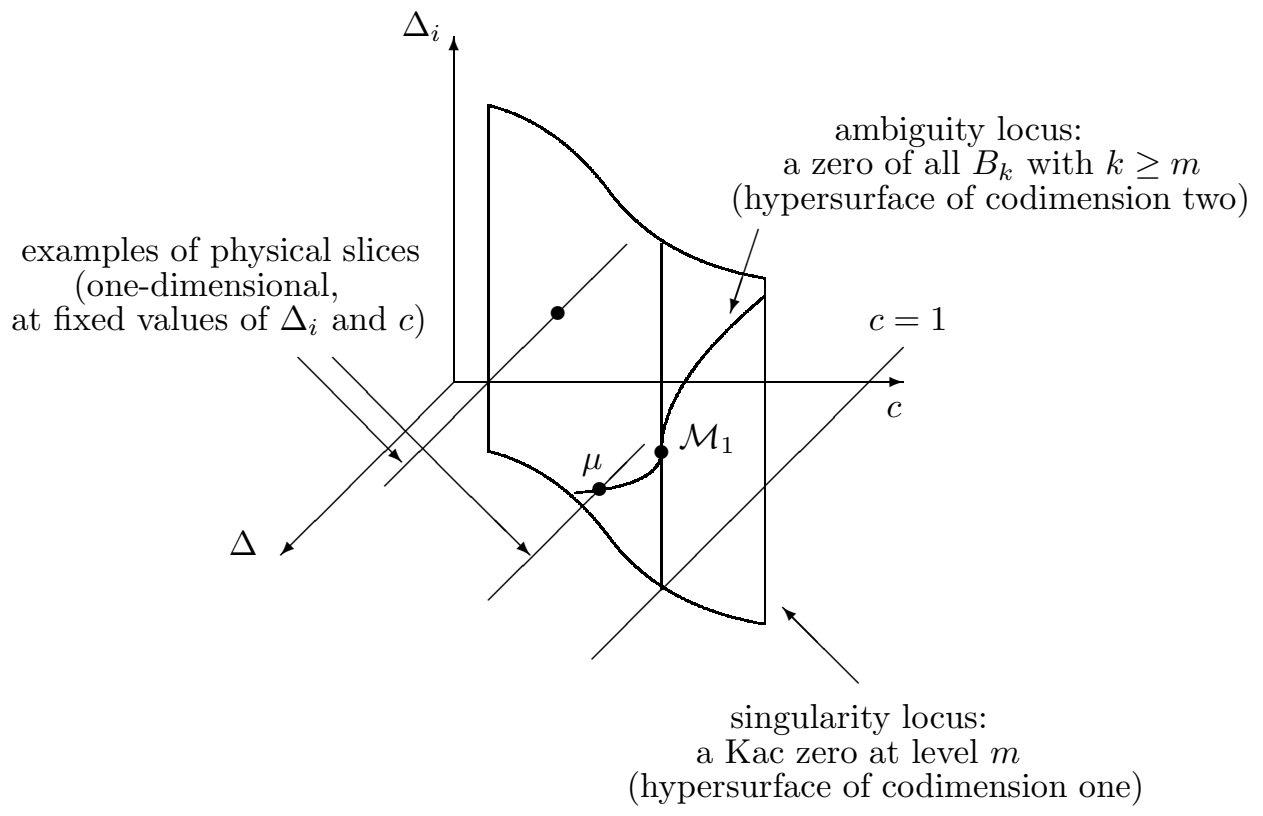

Of interest for us in this section will be the points $\mu \in \mathcal{A} \subset \mathcal{M}$, lying at the ambiguity locus $\mathcal{A}$. The thing is that most of interesting well known examples of conformal models, including Ashkin-Teller and minimal models are of this type. As we already saw in discussion of the vicinity of the point $\mu_{A T} \in \mathcal{M}_{1} \subset \mathcal{A} \subset \mathcal{M}$, all the coefficients $B_{k}$ can behave similarly near this locus, thus their common properties are inherited by the entire conformal block.

As before, at the vicinity of a point $\mu \in \mathcal{A}, \mu=\left\{\Delta_{i}^{(0)}, \Delta^{(0)}, c^{(0)}\right\}$ we put $\Delta_{i}=\Delta_{i}^{(0)}+\epsilon \delta_{i}, \Delta=\Delta^{(0)}+\epsilon \delta$, $c=c^{(0)}+\epsilon \sigma$ or $b=b^{(0)}+\epsilon \eta$ with a 6 -vector $\xi_{I}=\left(\delta, \delta_{1}, \ldots, \delta_{4}, \eta\right)$, and it turns out that

$$
B_{k}=b_{k}^{(0)}+d_{k} \cdot \frac{u_{I} \xi_{I}+O\left(\xi^{2}\right)}{v_{I} \xi_{I}+O\left(\xi^{2}\right)}
$$


if the zero is simple,

$$
B_{k}=b_{k}^{(0)}+d_{k} \cdot \frac{U_{I J} \xi_{I} \xi_{J}+O\left(\xi^{3}\right)}{V_{I J} \xi_{I} \xi_{J}+O\left(\xi^{3}\right)}
$$

if it is double zero etc. It often happens (as in the previous section) that

$$
\text { linear/quadratic forms } u, v \text { and } U, V \text { are independent of } k
$$

Note that the modified parametrization (4.21) is essential only if $c^{(0)}=1$, otherwise, $\sigma$ and $\eta$ are related linearly without any additional damping factor $\epsilon$.

Thus at the critical point we have both an ambiguity (the limit depends on the choice of direction to approach the point) and a universality, expressed by (5.6) and (5.9).

In the rest of this section we consider a few more examples of of (5.6) and (5.9).

\subsection{Phenomenon 1: nested structure of zeroes. $c=1$ example}

At $c=1$ all zeroes of the Kac determinants are doubled $\left(\Delta_{0}=\alpha^{2}\right.$ is itself a full square):

$$
\begin{gathered}
K_{2}=4 \Delta(4 \Delta-1)^{2}, \\
K_{3}=72 \Delta(4 \Delta-1)^{2}(\Delta-1)^{2}, \\
K_{4}=2304 \Delta^{2}(4 \Delta-1)^{2}(\Delta-1)^{2}(4 \Delta-9)^{2},
\end{gathered}
$$

The numerators of the coefficients $B_{k}$ at $c=1$ are nothing special. For instance, if one takes all the external dimensions equal to each other, $\Delta_{1}=\ldots=\Delta_{4}=\Delta_{e}$, the numerators of $B_{2}$ and $B_{3}$ are respectively

$$
N B_{2}=\Delta\left(\Delta-6 \Delta^{2}+9 \Delta^{3}+8 \Delta^{4}+16 \Delta_{e}^{2} \Delta-8 \Delta_{e} \Delta+8 \Delta_{e} \Delta^{2}+8 \Delta_{e}^{2}\right)
$$

and

$$
N B_{3}=3 \Delta(\Delta+2)(\Delta-1)^{2}\left(8 \Delta^{4}+19 \Delta^{3}+24 \Delta_{e} \Delta^{2}-11 \Delta^{2}+48 \Delta_{e}^{2} \Delta-24 \Delta_{e} \Delta+2 \Delta+24 \Delta_{e}^{2}\right)
$$

Things, however, change considerably if one looks at these numerators at the Kac zeroes. For example, at $\Delta=\alpha_{2,1}^{2}=\Delta_{2,1}(c=1)=\Delta_{1,2}(c=1)=\frac{1}{4}$,

$$
\begin{gathered}
N B_{2}=\frac{3}{256}\left(1-8\left(\Delta_{1}+\Delta_{2}\right)+16\left(\Delta_{1}^{2}+\Delta_{2}^{2}\right)-32 \Delta_{1} \Delta_{2}\right)\left(1-8\left(\Delta_{3}+\Delta_{4}\right)+16\left(\Delta_{3}^{2}+\Delta_{4}^{2}\right)-32 \Delta_{3} \Delta_{4}\right) \equiv \frac{3}{256} n b_{2} \\
N B_{3}=-\frac{27}{16384}\left(9+4\left(\Delta_{1}-\Delta_{2}\right)\right)\left(9+4\left(\Delta_{3}-\Delta_{4}\right)\right) \cdot n b_{2} \\
N B_{4} \sim n b_{2}, \\
\cdots \quad \\
N B_{k} \sim n b_{2} \quad \text { for } k \geq 2
\end{gathered}
$$

This is how (5.6) is realized in this case: any zero of $N B_{2}$ remains zero of higher $N B_{k}$.

Let us now look at the vicinity of a double zero of this $N B_{2}$. In this case $\Delta_{1}=\Delta_{2} \pm \sqrt{\Delta_{2}}+1 / 4 \Delta_{4}=$ $\Delta_{3} \pm \sqrt{\Delta_{3}}+1 / 4$ and one has (choosing for the sake of definiteness both signs plus):

$$
\begin{gathered}
B_{2}=\frac{\sqrt{\Delta_{2} \Delta_{3}}\left(4 \sqrt{\Delta_{2} \Delta_{3}}-2 \sqrt{\Delta_{2}}-2 \sqrt{\Delta_{3}}+3\right)}{4}+\frac{\sqrt{\Delta_{2} \Delta_{3}}\left(2 \sqrt{\Delta_{2}}+1\right)\left(2 \sqrt{\Delta_{3}}+1\right)}{4} r \\
B_{3}=\frac{\sqrt{\Delta_{2} \Delta_{3}}\left(20 \Delta_{2} \Delta_{3}+6 \Delta_{2} \sqrt{\Delta_{3}}+6 \Delta_{3} \sqrt{\Delta_{2}}+4 \Delta_{2}+4 \Delta_{3}+27 \sqrt{\Delta_{2} \Delta_{3}}-6 \sqrt{\Delta_{2}}-6 \sqrt{\Delta_{3}}+8\right)}{18} \\
+\frac{\left(\sqrt{\Delta_{2}}-2\right)\left(\sqrt{\Delta_{3}}-2\right)}{18} \frac{\sqrt{\Delta_{2} \Delta_{3}}\left(2 \sqrt{\Delta_{2}}+1\right)\left(2 \sqrt{\Delta_{3}}+1\right)}{4} r
\end{gathered}
$$


where

$$
r \equiv \frac{3 \eta^{2}-4 \delta\left(\hat{\delta}_{1}+\hat{\delta}_{2}+\hat{\delta}_{3}+\hat{\delta}_{4}\right)-6\left(\hat{\delta}_{1} \hat{\delta}_{3}+\hat{\delta}_{2} \hat{\delta}_{4}\right)+12 \hat{\delta}_{1} \hat{\delta}_{4}+3 \hat{\delta}_{2} \hat{\delta}_{3}}{4 \delta^{2}-9 \eta^{2}}
$$

and we introduced rescaled quantities: $\hat{\delta}_{1}=\delta_{1} /\left(2 \sqrt{\Delta_{2}}+1\right), \hat{\delta}_{2}=\delta_{2} / \sqrt{\Delta_{2}}, \hat{\delta}_{3}=\delta_{3} / \sqrt{\Delta_{3}}, \hat{\delta}_{4}=\delta_{4} /\left(2 \sqrt{\Delta_{3}}+1\right)$. This is how (5.9) works in the $c=1$ case.

Similarly, substituting $\Delta=\alpha_{3,1}^{2}=\Delta_{3,1}(c=1)=\Delta_{1,3}(c=1)=1$, one gets:

$$
\begin{gathered}
N B_{3}=-18\left(\Delta_{1}-\Delta_{2}\right)\left(1-2\left(\Delta_{1}+\Delta_{2}\right)+\left(\Delta_{1}-\Delta_{2}\right)^{2}\right)\left(\Delta_{3}-\Delta_{4}\right)\left(1-2\left(\Delta_{3}+\Delta_{4}\right)+\left(\Delta_{3}-\Delta_{4}\right)^{2}\right) \\
N B_{4}=100\left(4-\Delta_{1}+\Delta_{2}\right)\left(4+\Delta_{3}-\Delta_{4}\right) \cdot N B_{3}, \\
\cdots \\
N B_{k} \sim N B_{3} \quad \text { for } k \geq 3
\end{gathered}
$$

Thus, one observes that any zero of $N B_{3}$ remains zero of higher $N B_{k}$.

One can easily check that this remains true for other Kac zeroes.

\subsection{Phenomenon 2: nested structure is not always enhanced at multiple (irregu- lar) poles. $c=7 / 10$ and $c=1 / 2$ examples}

At $c=7 / 10$ there is a simple pole at $\Delta=3 / 2$ on the third level, i.e. at $B_{3}$, while in $B_{4}$ it becomes the double pole. This is because at $b=\sqrt{5} / 2$ there is an additional degeneracy: $\Delta_{1,3}=\Delta_{4,1}=\frac{3}{2}$.

When all $\Delta_{i}=3 / 2$, there is only a first-order zero in the numerators of both $B_{3}$ and $B_{4}$, so that $B_{4}$ remains infinite, while $B_{3}$ is just ambiguous, which is not like the cases we considered earlier. For instance, in the Ashkin-Teller case a non-zero multiplicity of the pole immediately resulted into the non-zero multiplicity of the corresponding zero of the conformal block numerator. This means that the in $c=7 / 10$ case the structure constants $C_{3 / 2,3 / 2}^{3 / 2}$ should vanish (while in the Ashkin-Teller case there is no need for this). In fact, this imposes restrictions on the rational conformal theories, since at rational values of the central charge there always emerge poles of higher multiplicities in the conformal block.

In $c=7 / 10$ theory the problem emerges in the symmetric point, when all the external dimensions are equal to each other. However, a similar phenomenon takes place already in a simpler $c=1 / 2$ theory though in a non-symmetric point. We now present this case in a little more detail.

At the central charge $c=1 / 2$ the Kac determinants are

$$
\begin{gathered}
K_{2}=2 \Delta_{0}\left(16 \Delta_{0}-1\right)\left(2 \Delta_{0}-1\right), \\
K_{3}=6 \Delta_{0}\left(16 \Delta_{0}-1\right)\left(2 \Delta_{0}-1\right)^{2}\left(3 \Delta_{0}-5\right), \\
K_{4}=6 \Delta_{0}\left(16 \Delta_{0}-1\right)^{2}\left(2 \Delta_{0}-1\right)^{2}\left(16 \Delta_{0}-21\right)\left(3 \Delta_{0}-5\right)\left(2 \Delta_{0}-7\right),
\end{gathered}
$$

The double zeroes in these formulas occur due to coincidence of the dimensions at $c=1 / 2: \Delta_{1,3}=\Delta_{2,1}=\frac{1}{2}$ and $\Delta_{2,2}=\Delta_{1,2}=\frac{1}{16}$. However, these accidental enhanced zeroes do not produce extra poles in $B_{3}$ and $B_{4}$ : for arbitrary values of the four external dimensions the numerator of $B_{3} \sim\left(2 \Delta_{0}-1\right)$, and $B_{4} \sim\left(16 \Delta_{0}-1\right)\left(2 \Delta_{0}-1\right)$, and this guarantees that the poles remain simple.

Nevertheless, the situation turns out to be not that simple. The remaining simple zero still needs to be compensated in the numerator, and now this is not universal. Indeed, let us consider the conformal block of $c=1 / 2$ theory with first two external dimensions parameterized as $\Delta_{1}=\frac{x^{2}-1}{48}+\frac{1}{3}+\frac{x}{6}$ and $\Delta_{2}=\frac{x^{2}-1}{48}$, which guarantees that the zero is simple in the denominator of $B_{2}$. However, in $B_{3}$ this does not provide an extra zero in addition to the factor $\left(2 \Delta_{0}-1\right)$ which emerges independently of the external dimensions. This breaks the nested structure of (5.6), and one has to impose an additional restriction to avoid infinities.

\subsection{Phenomenon 3: universality. $c=1 / 2$ example}

The leading behaviour is the vicinity of the singularity is

$$
B_{3}=\frac{(x+5)(x-1)(x+2)\left(\Delta_{3}-\Delta_{4}\right)\left(2 \Delta_{3}^{2}-4 \Delta_{3} \Delta_{4}+2 \Delta_{4}^{2}-3 \Delta_{3}-3 \Delta_{4}+1\right)}{3^{4} 2^{3}(3 \sigma-7 \delta)} \frac{1}{\epsilon}+O\left(\epsilon^{0}\right)
$$


Hence, one can either choose a particular $x$, or specially match $\Delta_{3}$ and $\Delta_{4}$.

In the first case, one can choose, for instance, $x=-2$. Then, the nested structure is restored: this condition is enough to cancel poles in $B_{3}, B_{4}, \ldots$ However, the universality (5.9) is broken down similarly to the Ashkin-Teller case in the previous section: the conformal block looks like

$$
\begin{gathered}
B_{1}=\frac{2 \Delta_{3}-\Delta_{4}+1}{4} \\
B_{2}=\frac{1}{7 \cdot 2^{5}}\left[36\left(\Delta_{3}^{2}+\Delta_{4}^{2}-\Delta_{3} \Delta_{4}\right)+88 \Delta_{3}-80 \Delta_{4}+31\right]+\frac{1}{7 \cdot 2^{5}}\left(12 \Delta_{3}^{2}-24 \Delta_{3} \Delta_{4}+12 \Delta_{4}^{2}-8 \Delta_{3}-8 \Delta_{4}+1\right) r \\
B_{24 \geq k \geq 3}=B_{k}^{(0)}+B_{k}^{(1)} r+B_{k}^{(2)} r_{2}
\end{gathered}
$$

since the pole at $\Delta=1 / 2$ becomes triple at level 25: $\Delta_{(5,5)}=1 / 2$ at $c=1 / 2$. Here

$$
\begin{gathered}
r \equiv \frac{\sigma-28 \delta_{1}-28 \delta_{2}}{7 \delta+2 \sigma} \\
r_{2} \equiv \frac{\delta_{1}-\delta_{2}}{3 \sigma-7 \delta}
\end{gathered}
$$

In the second case, for matching $\Delta_{3}$ and $\Delta_{4}$ one can use the same parametrization: $\Delta_{4}=\frac{y^{2}-1}{48}+\frac{1}{3}+\frac{y}{6}$ and $\Delta_{3}=\frac{y^{2}-1}{48}$ so that

$$
B_{3}=\frac{5}{3^{7} 2^{7}} \frac{(x+5)(x-1)(x+2)(y+5)(y-1)(y+2)}{3 \sigma-7 \delta} \frac{1}{\epsilon}+O\left(\epsilon^{0}\right)
$$

and one suffices to choose $y=1, y=-2$ or $y=-5$. However, in this case of two restricted dimensions and one matched to cancel the pole in the denominator, i.e. in the case of only one-parametric subspace in the 4-dimensional space $\left(\Delta_{1}, \Delta_{2}, \Delta_{3}, \Delta_{4}\right)$, one can observe a new phenomenon.

\subsection{Phenomenon 4: additional universality of minimal models. Ising model ex- ample}

In the case of the four external dimensions, parameterized by two variables $(x, y)$ as above (so that appropriate zero occurs in the numerator), the conformal block is equal at, say, $x=-2$ to

$$
\begin{gathered}
B_{1}=\frac{1-y}{2} \\
B_{2}=\frac{(y-1)(y-7)}{3 \cdot 2^{6}} \\
B_{3}=-\frac{5}{3^{4} 2^{8}}(y-1)(y-7)(y-13)-\frac{5}{3^{4} 2^{6}}(y-1)(y+2)(y+5) r \\
B_{4}=\frac{1}{7 \cdot 3^{5} 2^{13}}(y-1)\left(145 y^{3}-4575 y^{2}+58143 y-211825\right)+\frac{5}{3^{5} 2^{8}}(y-19)(y+2)(y+5)(y-1) r
\end{gathered}
$$

with

$$
r=\frac{\delta_{1}-\delta_{2}}{3 \sigma-7 \delta}
$$

One can see that with such a choice of the external dimensions, the coefficient $B_{2}$ is unambiguous, while the ambiguity goes away in higher $B_{k}$ as soon as one chooses $y=1,-2,-5$, since the coefficients in front of $r$ are cancelled in such a case. Note now that the external dimensions $\left(\Delta_{3}, \Delta_{4}\right)$ is $(0,1 / 2)$ at $y=1,(1 / 16,1 / 16)$ at $y=-2$ and $(1 / 2,0)$ at $y=-5$. These dimensions are exactly the ones of the Ising model: the model with the central charge $c=1 / 2$, and the three primary operators: with dimensions $0,1 / 2$ and $1 / 16$.

Thus, in the Ising model we encounter a new phenomenon: the coefficient in front of $r$ vanishes, i.e.

dependence on direction of approach to the Ising point disappears 
despite the Ising point lies on the ambiguity locus.

In fact, this remains the case for other minimal models (see an example of $c=1$ case in Appendix B), and thus (if there are no other examples of this kind) can serve as still another definition of minimal models, entirely at the level of perturbative conformal blocks.

Let us see how it works with various 4-point conformal blocks in the vicinity of the Ising point.

$\Delta_{e}^{(0)}=\frac{1}{16}, \Delta^{(0)}=\frac{1}{2}$

$$
\begin{gathered}
B_{2}=\frac{\frac{9}{32}(2 \sigma+7 \delta) \epsilon+O\left(\epsilon^{2}\right)}{2(2 \sigma+7 \delta) \epsilon+O\left(\epsilon^{2}\right)}=\frac{9}{64}+O(\epsilon), \\
B_{3}=\frac{\frac{75}{128}(2 \sigma+7 \delta)(3 \sigma-7 \delta) \epsilon^{2}+O\left(\epsilon^{3}\right)}{6(2 \sigma+7 \delta)(3 \sigma-7 \delta) \epsilon^{2}+O\left(\epsilon^{3}\right)}=\frac{25}{256}+O(\epsilon), \\
B_{4}=\frac{\frac{502047}{2048}(2 \sigma+7 \delta)(3 \sigma-7 \delta) \epsilon^{2}+O\left(\epsilon^{3}\right)}{12 \cdot 273 \cdot(2 \sigma+7 \delta)(3 \sigma-7 \delta) \epsilon^{2}+O\left(\epsilon^{3}\right)}=\frac{613}{8192}+O(\epsilon), \\
\ldots
\end{gathered}
$$

$\Delta_{e}^{(0)}=\frac{1}{16}, \Delta^{(0)}=0$

$$
\begin{gathered}
B_{2}=\frac{\frac{1}{32} \delta \epsilon+O\left(\epsilon^{2}\right)}{2 \delta \epsilon+O\left(\epsilon^{2}\right)}=\frac{1}{64}+O(\epsilon), \\
B_{3}=\frac{\frac{15}{32} \delta \epsilon+O\left(\epsilon^{2}\right)}{30 \delta \epsilon+O\left(\epsilon^{2}\right)}=\frac{1}{64}+O(\epsilon), \\
B_{4}=\frac{-\frac{257985}{4096} \delta \epsilon+O\left(\epsilon^{2}\right)}{-4410 \delta \epsilon+O\left(\epsilon^{2}\right)}=\frac{117}{8192}+O(\epsilon)
\end{gathered}
$$

again there is no $r$-dependence.

See more details about the Ising model in Appendix A.

\subsection{Summary}

In this section we originated a detailed examination of Kac zeroes, where most of conventionally studied conformal models are located, and where the standard near-divisor ambiguity arises, preventing definition of conformal blocks, both perturbative and non-perturbative, as the well-defined limit from non-singular expressions. We saw in the previous section 4 that this ambiguity can explain the apparent difference between available non-perturbative conformal blocks at the Ashkin-Teller point, and thus we believe that understanding of the near-divisor structure will be important for further development of non-perturbative CFT. As we explained, already at the first glance, this structure is quite interesting. Namely, we described four non-trivial phenomena specific for conformal blocks and emphasizing that they are far from exhibiting a generic behavior near the singularity: quite the opposite, their behavior is adjusted in a very special way, which should be better studied and interpreted.

So, when we look at the Kac zero at level $l$, the corresponding singularity appears first in the coefficient $B_{l}$, and to avoid singularity one should adjust the external dimensions $\Delta_{i}$ to make the numerator of $N B_{l}$ vanishing as well. Then the pole continues to be present in all the higher coefficients $B_{k}$ with $k \geq l$. But:

- Phenomenon 1. There is a nested structure in conformal blocks: the zero of the numerator $N B_{l}$ at this pole is also present in all higher numerators $N B_{k}$ with $k \geq l$. This means that when the Kac zero is simple, it is enough to adjust external dimensions only once, in the first relevant $B_{l}$, and then the entire conformal block is non-singular: the zero of the first relevant numerator at the simple Kac zero is inherited by all other numerators.

However, for many interesting choices of internal (intermediate) dimension $\Delta$, the zero of Kac determinant is not simple, there are "accidental" coincidences of different zeroes in the numerators of $B_{k}$ with $k \geq l$ for all conformal theories with rational central charges. Then 
- Phenomenon 2. The nested structure gets broken, in the sense that the zero in the numerator of conformal block remains simple. Thus, for accidentally degenerate Kac zeroes we encounter "naked singularities" like in generic function on the moduli space.

However, there is a notable exception from this pessimistic picture:

- Phenomenon 3. The nested structure is typically restored, as soon as one additionally adjusts the cancellation of the multiple pole in the first relevant $B_{l}$. In this case, the intermediate $\Delta$ is such that the Kac zeroes are not simple, but one and the same choice of external $\Delta_{i}$ provides all the numerators with the zeroes of exactly the right order to eliminate the singularity. Then, the ambiguity in the conformal block is piece-wise universal: if the simple Kac zero emerges at some $l_{1}$, it becomes a double zero at some $l_{2}$ etc (as soon as there emerges an "accidental" double pole, sooner or later there emerge all higher multiplicities), all $B_{k<l_{1}}$ are unambiguous, $B_{l_{1} \leq k<l_{2}}$ are universal linear functions of one parameter describing approach to the singularity locus, $B_{l_{2} \leq k<l_{3}}$ are universal functions which are linear combinations of two parameters etc.

More than that:

- Phenomenon 4. At the minimal model points the near-divisor ambiguity disappears: the limit does not depend on the direction on the moduli space, from which we approach the minimal model points. This is probably the most spectacular manifestation of how special the minimal models really are from the point of view of conformal block properties, and relation of this property to many others (like the finiteness of block quantity, needed for the conformal bootstrap at these points) still remains to be understood.

It is not clear if these four phenomena provide an exhaustive description of peculiarities of the conformal block behavior at the Kac divisor, even in the simplest 4-point spherical case. Very interesting should be extension of this study to more points and higher genera. And, of course, the crucial question is the implication for non-perturbative corrections. All this remains to be thoroughly investigated.

\section{Null-vectors, equations and hidden parameters}

In the previous sections we studied ambiguities that appear at the singularity locus of the conformal block and clarified their origin and peculiar properties. Here we consider the specific conformal blocks when some of the external dimensions correspond to a degenerate vector, since in this case one can deal with the conformal block not as a series but a space of solutions to a differential equation. Hence, one can check if it is possible to find some non-perturbative hidden parameters, i.e. if a conformal block can be presented as a linear combination of solutions with some arbitrary coefficients.

We start the simplest example of the vector degenerate at the second level. This vector is of the form $\tilde{V}=\left(\xi L_{-1}^{2}-L_{-2}\right) V_{\Delta}$ and there are two non-trivial conditions: $L_{1} \tilde{V}=0$ and $L_{2} \tilde{V}=0$. They imply respectively that

$$
\xi=\frac{3}{2(2 \Delta+1)}
$$

and

$$
8 \Delta+c=12 \xi \Delta
$$

or, together

$$
\Delta=\frac{5-c \pm \sqrt{(c-1)(c-25)}}{16}
$$

Parameterizing the central charge and dimension as in (3.26), we obtain four solutions:

$$
\left\{\begin{array}{c}
\alpha=\frac{1}{2 b} \\
\xi=b^{2}
\end{array}, \quad\left\{\begin{array}{c}
\alpha=-\frac{b}{2} \\
\xi=\frac{1}{b^{2}}
\end{array}, \quad\left\{\begin{array}{c}
\alpha=\frac{3 b}{2}-\frac{1}{b} \\
\xi=b^{2}
\end{array}, \quad\left\{\begin{array}{c}
\alpha=b-\frac{3}{2 b} \\
\xi=\frac{1}{b^{2}}
\end{array}\right.\right.\right.\right.
$$

In what follows we work with the first of these four solutions (boxed), so that the original highest weight primary $V_{1 / 2 b}$ of degenerate Verma module has dimension

$$
\Delta_{1 / 2 b}=-\frac{1}{2}+\frac{3}{4 b^{2}}
$$


The conformal Ward identities imply that 4-point correlators $\Psi_{4}(x, \bar{x})$ with insertion of this degenerate primary at point $x$ satisfy peculiar differential equations, see [1]:

$$
\left\{b^{2} x(x-1) \partial_{x}^{2}+(2 x-1) \partial_{x}+\Delta_{1 / 2 b}+\frac{\Delta_{1}}{x}-\frac{\Delta_{3}}{x-1}-\Delta_{4}\right\} \Psi_{4}(x)=0
$$

where we suppressed the dependence on $\bar{x}$. In the free field realization of conformal field theory this constraint is imposed almost automatically, see [61], and this is also easily seen from the Dotsenko-Fateev $\beta$-ensemble representation of the corresponding conformal blocks, [61].

Conjugation with a factor $x^{\alpha}(1-x)^{\beta}$ with specially adjusted $\alpha$ and $\beta$ converts (6.6) into an ordinary hypergeometric equation with the solution

$$
\begin{gathered}
\Psi_{4}(x)=x^{\alpha_{1} / b}(1-x)^{\alpha_{3} / b} F(A, B ; C ; x) \\
A=\frac{1}{2 b^{2}}+\frac{\alpha_{1}}{b}+\frac{\alpha_{2}}{b}-\frac{\alpha_{3}}{b} \\
B=\frac{1}{b} \sum_{i=1}^{3} \alpha_{i}+2 \Delta_{1 / 2 b}, \quad C=\frac{1}{b^{2}}+\frac{2 \alpha_{1}}{b}
\end{gathered}
$$

Equations (6.6), (6.7) are consistent with generic formulas (3.22)-(3.23) only if the dimensions $\Delta_{1}$ and $\Delta$ are related (in parametrization $(3.26)$ ) by the fusion rule

$$
\alpha=\alpha_{1} \pm \frac{1}{2 b}
$$

where two choices of the sign correspond to the two linearly independent solutions of (6.6) and in the case of the sign "minus" in (6.8) one has to choose in (6.7) instead of $F(A, B ; C ; x)$ the other solution to the hypergeometric equation so that $\alpha_{1} \rightarrow b-1 / b-\alpha_{1}$ in $\Psi_{4}(x)$ in (6.7).

One can easily check directly that the conformal block from the r.h.s. of (6.10)

$$
\mathcal{B}_{\Delta_{\alpha}}^{(1,1 / 2 b ; 34)}(x)=x^{\Delta_{\alpha}-\Delta_{1}-\Delta_{1 / 2 b}}\left(1+\frac{\left(\Delta_{\alpha}+\Delta_{1 / 2 b}-\Delta_{1}\right)\left(\Delta_{\alpha}+\Delta_{3}-\Delta_{4}\right)}{2 \Delta_{\alpha}} x+\ldots\right) \stackrel{(6.8)}{=} \Psi_{4}(x)
$$

which solves (6.6). Formula (3.1) now acquires the form

$$
\begin{gathered}
\left\langle V_{1}(0) V_{1 / 2 b}(x) V_{3}(1) V_{4}(\infty)\right\rangle=\sum_{\Delta, \bar{\Delta}} C_{1,1 / 2 b}^{\Delta, \bar{\Delta}} C_{34}^{\Delta, \bar{\Delta}} \mathcal{B}_{\Delta}^{(1,1 / 2 b ; 34)}(x) \overline{\mathcal{B}}_{\bar{\Delta}}^{(1,1 / 2 b ; 34)}(\bar{x})= \\
=\sum_{\substack{\alpha=\alpha_{1} \pm \frac{1}{2 b} \\
\bar{\alpha}=\bar{\alpha}_{1} \pm \frac{1}{2 b}}} C_{1,1 / 2 b}^{\Delta_{\alpha}, \Delta_{\bar{\alpha}}} C_{34}^{\Delta_{\alpha}, \Delta_{\bar{\alpha}}} \mathcal{B}_{\Delta}^{(1,1 / 2 b ; 34)}(x) \overline{\mathcal{B}}_{\bar{\Delta}}^{(1,1 / 2 b ; 34)}(\bar{x})
\end{gathered}
$$

since only for the choice (6.8) the structure constant $C_{1,1 / 2 b}^{\Delta_{\alpha}}$ is non-vanishing [1]. Here we obtained this fact indirectly by solving the equation for the correlator. One can derive this fact straightforwardly using the $\beta$-ensemble representation for the conformal blocks [61].

Thus, two solutions of the equation for the degenerate conformal block describes two different conformal blocks, the only two with non-zero structure constants. One can easily see also (6.8) from their asymptotics: since the conformal block behaves at small $x$ like $x^{\Delta-\Delta_{1}-\Delta_{2}}$, one gets (6.8) from the asymptotic behaviours $\Psi_{4}^{(1)}(x) \sim x^{\alpha_{1} / b}$ and $\Psi_{4}^{(2)}(x) \sim x^{1-1 / b^{2}-\alpha_{1} / b}$.

This system of two conformal blocks is self-consistent: it survives the modular transformation (3.3): $x \rightarrow$ 
$1-x, \Delta_{1} \leftrightarrow \Delta_{3}$. Indeed (see $\left.[60,(9.131(1,2))]\right)$,

$$
\begin{gathered}
\Psi_{4}^{(1)}\left(\Delta_{3}, \Delta_{2}, \Delta_{1}, \Delta_{4} ; 1-x\right)=\frac{\Gamma\left(\frac{2 \alpha_{3}}{b}+\frac{1}{b^{2}}\right) \Gamma\left(\frac{1}{b^{2}}+\frac{2 \alpha_{1}}{b}-1\right)}{\Gamma\left(\frac{3}{2 b^{2}}-1+\frac{\alpha_{1}}{b}+\frac{\alpha_{3}}{b}+\frac{\alpha_{4}}{b}\right) \Gamma\left(\frac{1}{2 b^{2}}+\frac{\alpha_{1}}{b}+\frac{\alpha_{3}}{b}-\frac{\alpha_{4}}{b}\right)} \Psi_{4}^{(1)}\left(\Delta_{1}, \Delta_{2}, \Delta_{3}, \Delta_{4} ; x\right)+ \\
+\frac{\Gamma\left(\frac{2 \alpha_{3}}{b}+\frac{1}{b^{2}}\right) \Gamma\left(1-\frac{1}{b^{2}}-\frac{2 \alpha_{1}}{b}\right)}{\Gamma\left(1-\frac{1}{2 b^{2}}+\frac{\alpha_{3}}{b}-\frac{\alpha_{1}}{b}-\frac{\alpha_{4}}{b}\right) \Gamma\left(\frac{1}{2 b^{2}}+\frac{\alpha_{3}}{b}+\frac{\alpha_{4}}{b}-\frac{\alpha_{1}}{b}\right)} \Psi_{4}^{(2)}\left(\Delta_{1}, \Delta_{2}, \Delta_{3}, \Delta_{4} ; x\right) \\
\Psi_{4}^{(2)}\left(\Delta_{3}, \Delta_{2}, \Delta_{1}, \Delta_{4} ; 1-x\right)=\frac{\Gamma\left(2-\frac{2 \alpha_{3}}{b}-\frac{1}{b^{2}}\right) \Gamma\left(\frac{1}{b^{2}}-1+\frac{2 \alpha_{1}}{b}\right)}{\Gamma\left(\frac{1}{2 b^{2}}+\frac{\alpha_{1}}{b}+\frac{\alpha_{4}}{b}-\frac{\alpha_{3}}{b}\right) \Gamma\left(1-\frac{1}{2 b^{2}}+\frac{\alpha_{1}}{b}-\frac{\alpha_{3}}{b}-\frac{\alpha_{4}}{b}\right)} \Psi_{4}^{(1)}\left(\Delta_{1}, \Delta_{2}, \Delta_{3}, \Delta_{4} ; x\right)+ \\
\Gamma\left(2-\frac{2 \alpha_{3}}{b}-\frac{1}{b^{2}}\right) \Gamma\left(1-\frac{1}{b^{2}}-\frac{2 \alpha_{1}}{b}\right) \\
+\frac{\left.\alpha_{4}\right) \Gamma\left(1-\frac{1}{2 b^{2}}+\frac{\alpha_{1}}{b}+\frac{\alpha_{3}}{b}-\frac{\alpha_{4}}{b}\right)}{\Gamma\left(2-\frac{3}{2 b^{2}}-\frac{\alpha_{1}}{b}-\frac{\alpha_{3}}{b}-\frac{\alpha_{4}}{b}\right)} \Psi_{4}^{(2)}\left(\Delta_{1}, \Delta_{2}, \Delta_{3}, \Delta_{4} ; x\right)
\end{gathered}
$$

i.e. the modular kernel in this case is the $2 \times 2$ matrix

$$
\left(\begin{array}{cc}
\frac{\Gamma\left(\frac{2 \alpha_{3}}{b}+\frac{1}{b^{2}}\right) \Gamma\left(\frac{1}{b^{2}}+\frac{2 \alpha_{1}}{b}-1\right)}{\Gamma\left(\frac{3}{2 b^{2}}-1+\frac{\alpha_{1}}{b}+\frac{\alpha_{3}}{b}+\frac{\alpha_{4}}{b}\right) \Gamma\left(\frac{1}{2 b^{2}}+\frac{\alpha_{1}}{b}+\frac{\alpha_{3}}{b}-\frac{\alpha_{4}}{b}\right)} & \frac{\Gamma\left(\frac{2 \alpha_{3}}{b}+\frac{1}{b^{2}}\right) \Gamma\left(1-\frac{1}{b^{2}}-\frac{2 \alpha_{1}}{b}\right)}{\Gamma\left(1-\frac{1}{2 b^{2}}+\frac{\alpha_{3}}{b}-\frac{\alpha_{1}}{b}-\frac{\alpha_{4}}{b}\right) \Gamma\left(\frac{1}{2 b^{2}}+\frac{\alpha_{3}}{b}+\frac{\alpha_{4}}{b}-\frac{\alpha_{1}}{b}\right)} \\
\frac{\Gamma\left(2-\frac{2 \alpha_{3}}{b}-\frac{1}{b^{2}}\right) \Gamma\left(\frac{1}{b^{2}}-1+\frac{2 \alpha_{1}}{b}\right)}{\Gamma\left(\frac{1}{2 b^{2}}+\frac{\alpha_{1}}{b}+\frac{\alpha_{4}}{b}-\frac{\alpha_{3}}{b}\right) \Gamma\left(1-\frac{1}{2 b^{2}}+\frac{\alpha_{1}}{b}-\frac{\alpha_{3}}{b}-\frac{\alpha_{4}}{b}\right)} & \frac{\Gamma\left(2-\frac{2 \alpha_{3}}{b}-\frac{1}{b^{2}}\right) \Gamma\left(1-\frac{1}{b^{2}}-\frac{2 \alpha_{1}}{b}\right)}{\Gamma\left(2-\frac{3}{2 b^{2}}-\frac{\alpha_{1}}{b}-\frac{\alpha_{3}}{b}-\frac{\alpha_{4}}{b}\right) \Gamma\left(1-\frac{1}{2 b^{2}}+\frac{\alpha_{1}}{b}+\frac{\alpha_{3}}{b}-\frac{\alpha_{4}}{b}\right)}
\end{array}\right)
$$

We demonstrate how all this works in the minimal models in examples of $c=1 / 2$ (the Ising model) and $c=1$ in Appendices A and B respectively.

Similarly one can deal with conformal blocks degenerate at higher levels. In these case the order of the corresponding differential equation is higher, but again is equal exactly to the number of conformal blocks with non-zero structure constants. The modular matrix also accordingly increases its size, see examples in [1]. Hence, no hidden parameters are emerge in this way. In fact, this is not surprising, since the differential equations are w.r.t. the variable $x$, and, as we already stressed, the $x$-behaviour of the conformal block is not expected to depend on non-perturbative (hidden) parameters.

\section{Conclusion}

The main goal of this paper is to urge the study of the non-perturbative conformal block as a function of all its variables: coordinates, external and internal dimensions and the central charge. The first question to ask here is if there are some extra parameters, besides already enumerated, on which the non-perturbative quantity usually depends, which are not seen at the perturbative level like the theta-angle in instanton calculus. In conformal block story, the main ambiguity is the overall normalization, which can be a function of dimensions and central charge (thus in fact can contain infinitely many extra parameters). The lack of control over such normalization factors is the main current problem in relating different efficient non-perturbative approaches, say, to constructing the modular kernel: the $S L_{q}(2)$ method of Ponsot-Teshner [41], the matrix model approach of [57] uncovering the Stokes (wall crossing) phenomena and relating cluster variables to check-exponents of [62], and the Painleve equation method of [59]. In the present paper we did not attack this problem of $\Delta$-dependence directly: instead we tried to look for extra non-perturbative parameters, considering the $x$-dependence of the simplest (4-point spherical) conformal block. We looked mostly at two obvious places: at discrepancy between the explicitly known non-perturbative Zamolodchikov and elliptic-integral answers at the Ashkin-Teller point (they are different functions of $x$ ), and at the higher order differential equation in $x$, which conformal block satisfies when one of the vertex operators is degenerate (e.g. in the Ising and other minimal models), both cases could seem to imply the existence of extra parameters. As we explained, this is, however, not the case. The discrepancy at the Ashkin-Teller point (and in many similar cases) is in fact just the ordinary ambiguity at the singularity divisor for a function of many variables (dimensions and central charge), and no extra parameters are present. The case with many, rather than one, solutions to a higher order differential equation is resolved not by introduction of extra variables, but by the fact that $B(x)$ actually does not satisfy such equations when just one 
external dimension is fixed: in fact, the equation is true for the conformal block only when the internal dimension $\Delta$ is fixed as well [61] (this is a very important feature of the conformal block, which is often overlooked or underestimated). In result, the extra solutions are in fact describing not an ambiguity in the function $B_{\Delta}(x)$, but the other conformal blocks $B_{\Delta^{\prime}}(x)$ with the other allowed values of internal dimension $\Delta^{\prime}$ : there exactly as many of them as the degeneration level of vertex operator and the order of the differential equation.

Thus we found no evidence for extra non-perturbative parameters in the $x$-dependence of conformal block. This could seem obvious from the very beginning: as we already mentioned, there is a belief that the 4-point spherical conformal block is actually a Belyi function (i.e. has only non-essential singularities at three points $x=0,1, \infty$, and ramification orders are integer for rational conformal models). This, in turn, can be attributed either to the fact that $2 d$ CFT is actually a free field theory [1] so that there is actually no interaction and no reason for real non-perturbative effects to exist, or to another fact: that it is conformal, and then no non-trivial dependence is allowed for a function of the single dimensional parameter $x$.

We believe that our simple consideration sheds some new light on the problem of non-perturbative conformal blocks and can help to attract new attention to this extremely interesting problem. Conformal blocks are the crucially important special function of the string era, and they should be thoroughly investigated and understood.

\section{Acknowledgements}

Our work is partly supported by the grant NSh-1500.2014.2 (A.M.'s), by RFBR 13-02-00457 (A.Mir.), 13-0200478 (A.Mor.), by joint grants 13-02-91371-ST (A.M.'s), 14-01-92691-Ind (A.M.'s), by the Brazil National Counsel of Scientific and Technological Development (A.Mor.). The research of H.I. is supported in part by the Grant-in-Ad for Scientific Research (23540316) from the Ministry of Education, Science and Culture, Japan. Support from JSPS/RFBR bilateral collaboration Synthesis of integrabilities arising from gauge-string duality (FY2010-2011: 12-02-92108-Yaf-a) is gratefully appreciated.

\section{Appendix A. Ising model}

The critical behaviour of the Ising model is described by the central charge $c=1 / 2$, and we choose $b=\sqrt{3} / 2$. There three primary fields with the dimensions: $\Delta_{I}=0$ (i.e. $\alpha_{I}=0$ or $-\sqrt{3} / 6$ ), $\Delta_{\psi}=1 / 2$ (i.e. $\alpha_{\psi}=\sqrt{3} / 3$ or $-\sqrt{3} / 2$ ), $\Delta_{\sigma}=1 / 16$ (i.e. $\alpha_{\sigma}=\sqrt{3} / 12$ or $-\sqrt{3} / 4$ ). The first field is degenerate at the first level, the second and the third ones are degenerate at the second level. One can calculate the conformal blocks in different cases. For instance, consider $\mathcal{B}\left(\Delta_{1}, \Delta_{2}, \Delta_{3}, \Delta_{4} ; \Delta ; c ; x\right)$ and suppose that the intermediate dimension $\Delta=\Delta_{\psi}=1 / 2$. It leads to poles at all levels higher than one. The condition of canceling this pole by matching the dimensions $\Delta_{1}$ and $\Delta_{2}$ requires them be either $1 / 2$ and 0 or $\Delta_{1}=\Delta_{2}=1 / 16$ (if one restricts himself with the spectrum of three fields above). Hence, the correlators of fields $\langle\psi \psi I\rangle$ and $\langle\psi \sigma \sigma\rangle$ are non-zero. Similarly considering the conformal block with the intermediate dimension $\Delta=\Delta_{\sigma}=1 / 16$, one finds the non-zero correlator $\langle\psi \psi I\rangle$. This fixes the operator product expansion (OPE) of fields.

Now consider the conformal block with the field $\psi(x)$ at point $x$ (remind that, in our notation, this corresponds to $\Delta_{2}$, while $\Delta_{1}, \Delta_{3}$ and $\Delta_{4}$ corresponds to the fields at points 0,1 and $\infty$ respectively). This field is degenerate at the second level and the conformal block satisfies a second order differential equation provided $\alpha=\alpha_{1} \pm 1 / 2 b$ (see s.6). This condition can be satisfied only for the pairs $\left(\alpha, \alpha_{1}\right)$ either $\left(\alpha_{\sigma}, \alpha_{\sigma}\right)$ or $\left(\alpha_{\psi}, \alpha_{I}\right)$ which again fixes the OPE. Consider the correlator $\langle\sigma(0) \psi(x) \sigma(1) \psi(\infty)>$. It is described by the values $\alpha_{1}=-\sqrt{3} / 4, \alpha_{2}=\sqrt{3} / 3, \alpha_{3}=-\sqrt{3} / 4, \alpha_{4}=-\sqrt{3} / 2$. The corresponding conformal block $\mathcal{B}(x)$ satisfies the differential equation (6.6)

$$
\left[\frac{3}{4} x(1-x) \partial_{x}^{2}+(2 x-1) \partial_{x}+\frac{1}{16}\left(\frac{1}{x}+\frac{1}{1-x}\right)\right] \mathcal{B}(x)=0
$$

This equation is hypergeometric and has two solutions:

$$
\begin{gathered}
\mathcal{B}^{(1)}(x)=\frac{1-2 x}{\sqrt{x(1-x)}} \\
\mathcal{B}^{(2)}(x)=[x(1-x)]^{1 / 6} F(1 / 3,2 ; 5 / 3 ; x)
\end{gathered}
$$

where $F(a, b ; c ; x)$ is the hypergeometric function. The first solution corresponds to the behaviour at small $x$

$$
\mathcal{B}^{(1)}(x)=\frac{1}{\sqrt{x}}\left(1-\frac{3}{2} x-\frac{5}{8} x^{2}-\frac{7}{16} x^{3}-\frac{45}{128} x^{4}+\ldots\right)
$$


of the conformal block. The multiplier $1 / \sqrt{x}$ comes from the usual pre-factor $x^{\Delta-\Delta_{1}-\Delta_{2}}$ of the conformal block and implies (which we already established from the OPE earlier) that $\Delta=\Delta_{\sigma}=1 / 16$. The second solution ought to describe an intermediate field with dimension $\Delta=35 / 48$. This field is absent in the spectrum which means that the corresponding structure constant is zero.

The expansion (A3) of the conformal block should be compared with its generic expansion (3.21). One can check that they coincide independently on the way the singularity is resolved, i.e. if one considers a vicinity of the point $\Delta_{1}=1 / 16, \Delta_{2}=1 / 2, \Delta_{3}=16, \Delta_{4}=1 / 2, \Delta=1 / 16$, the leading order in $\epsilon$ does not depend on the way of approaching the singularity at all (see s.5.6):

$$
(3.21)=1-\frac{3}{2} x-\frac{5}{8} x^{2}-\frac{7}{16} x^{3}-\frac{45}{128} x^{4}+\ldots
$$

Note that $\mathcal{B}^{(1)}(x)$ is consistently invariant with respect to the duality transformation $x \rightarrow 1-x$. Similarly invariant is the differential equation (A1), though the second solution is not: it transforms through itself and the first solution $[60,9.131(2)]$ :

$$
F(1 / 3,2 ; 5 / 3 ; x)=F(1 / 3,2 ; 5 / 3 ; 1-x)+\frac{\Gamma(2 / 3)^{3}}{\sqrt{3} \pi} \frac{1}{\left[x(1-x]^{1 / 6}\right.} \mathcal{B}^{(1)}(x)
$$

This means that the duality matrix is triangle.

Now consider another possible 4-point correlator in this theory: $\langle\sigma \sigma \sigma \sigma\rangle$. One can similarly right down the differential equation

$$
\left[\frac{4}{3} x(1-x) \partial_{x}^{2}+(2 x-1) \partial_{x}+\frac{1}{16}\left(\frac{1}{x}+\frac{1}{1-x}\right)\right] \mathcal{B}(x)=0
$$

This equation has two solutions:

$$
\begin{aligned}
\mathcal{B}^{(1)}(x) & =\frac{\sqrt{\sqrt{x}+1}+\sqrt{\sqrt{x}-1}}{\left[x(1-x]^{1 / 8}\right.} \\
\mathcal{B}^{(2)}(x) & =\frac{\sqrt{\sqrt{x}+1}-\omega \sqrt{\sqrt{x}-1}}{\left[x(1-x]^{1 / 8}\right.}
\end{aligned}
$$

where $\omega \equiv \exp ( \pm \pi i / 2)$ is a square root of -1 (plus or minus depends on the chosen branch of $\sqrt{x}$ ). The first solution has the small- $x$ expansion

$$
\mathcal{B}^{(1)}(x) \sim \frac{1}{x^{1 / 8}}\left(1+\frac{1}{64} x^{2}+\frac{1}{64} x^{3}+\frac{117}{8192} x^{4}+\frac{53}{4096} x^{5}+\ldots\right)
$$

and corresponds to the intermediate field $I$ with dimension $\Delta=\Delta_{I}=0$. The second solution has the small- $x$ expansion

$$
\mathcal{B}^{(2)}(x) \sim \frac{\sqrt{x}}{x^{1 / 8}}\left(1+\frac{1}{4} x+\frac{9}{64} x^{2}+\frac{25}{256} x^{3}+\frac{613}{8192} x^{4}\right)
$$

These two expansions as before reproduce the correct result (A3) independently on the way of resolving the singularity.

Since, in this case, the both solutions correspond to the "physical" conformal blocks, this is not surprising that the duality transformation acts as a matrix on these two solutions:

$$
\begin{aligned}
& \mathcal{B}^{(1)}(1-x)=\frac{1}{\sqrt{2}}\left(\mathcal{B}^{(1)}(x)+\frac{\omega}{2} \mathcal{B}^{(2)}(x)\right) \\
& \mathcal{B}^{(2)}(1-x)=\sqrt{2}\left(\mathcal{B}^{(1)}(x)-i \frac{\omega}{2} \mathcal{B}^{(2)}(x)\right)
\end{aligned}
$$

i.e. the duality matrix is

$$
S=\sqrt{2}\left(\begin{array}{cc}
1 & \frac{\omega}{2} \\
2 & -i \omega
\end{array}\right)
$$

The last non-trivial correlator in the Ising model is $\langle\psi \psi \psi \psi\rangle$. It is described by the differential equation

$$
\left[\frac{3}{4} x(1-x) \partial_{x}^{2}+(2 x-1) \partial_{x}+\frac{1}{2}\left(\frac{1}{x}+\frac{1}{1-x}\right)\right] \mathcal{B}(x)=0
$$


Again, only one of the two solutions of this equation is relevant to the Ising model, it is

$$
\mathcal{B}(x)=\frac{1-x+x^{2}}{x(1-x)}
$$

This solution is modular invariant and its small- $x$ expansion gives the conformal block (A3) independently on the way of resolving the singularity:

$$
\mathcal{B}(x) \sim \frac{1}{x}\left(1+x^{2}+x^{3}+x^{4}+\ldots\right)
$$

where the common factor $1 / x$ implies that this conformal block describes the intermediate field of dimension $\Delta=\Delta_{I}=0$ as it should be.

Appendix B. $c=1, \Delta_{e}=\frac{1}{4}$

Let us consider the minimal model with $c=1$, it can be obtained from the series of minimal models $(m, m+1)$ in the limit $m \rightarrow \infty$ [46, App.B], and one easily construct the conformal block of four fields with $\Delta_{(1,2)}=1 / 4$, since they are degenerate at the second level. Solving equation (6.6) with $b=1$ gives two solutions which correspond to the conformal block with internal dimensions $\Delta=0$ :

$$
\mathcal{B}_{\Delta=0}\left(\Delta_{i}=\frac{1}{4}, c=1 \mid x\right)=\frac{1}{\sqrt{x}}\left(1+\frac{x^{2}}{8}+\frac{x^{3}}{8}+\frac{15 x^{4}}{128}+\ldots\right)=\frac{1}{2 \sqrt{x}}\left(\sqrt{1-x}+\frac{1}{\sqrt{1-x}}\right)
$$

and $\Delta=1$

$$
\mathcal{B}_{\Delta=1}\left(\Delta_{i}=\frac{1}{4}, c=1 \mid x\right)=\sqrt{x}\left(1+\frac{x}{2}+\frac{3 x^{2}}{8}+\frac{5 x^{3}}{16}+\frac{35 x^{4}}{128}+\ldots\right)=\frac{\sqrt{x}}{\sqrt{1-x}}
$$

The modular transformation $x \longrightarrow 1-x$ acts on the doublet

$$
\left(\begin{array}{c}
\frac{1}{2} \sqrt{\frac{1-x}{x}}+\frac{1}{2} \sqrt{\frac{1}{x(1-x)}} \\
\sqrt{\frac{x}{1-x}}
\end{array}\right)
$$

by the matrix

$$
\left(\begin{array}{cc}
1 / 2 & 3 / 4 \\
1 & -1 / 2
\end{array}\right)
$$

From bilinear combination of these one can construct a modular invariant by adjusting the coefficient:

$$
\frac{1}{|x|}\left|\mathcal{B}_{0}\right|^{2}+\frac{3}{4}|x| \cdot\left|\mathcal{B}_{1}\right|^{2}=\frac{\left|1-\frac{x}{2}\right|^{2}+\frac{3}{4} \cdot|x|^{2}}{|x| \cdot|1-x|} \sim \frac{|x|}{|1-x|}+\frac{|1-x|}{|x|}+\frac{1}{|x(1-x)|}
$$

This answer coincides with [46].

\section{References}

[1] A.Belavin, A.Polyakov, A.Zamolodchikov, Nucl.Phys. B241 (1984) 333-380;

A.Zamolodchikov and Al.Zamolodchikov, Conformal field theory and critical phenomena in 2d systems, 2009 (in Russian);

L. Alvarez-Gaume, Helvetica Physica Acta, 64 (1991) 361;

P. Di Francesco, P. Mathieu, D. Senechal, Conformal Field Theory, Springer, 1996

[2] M.Sato, RIMS Kokyuroku, 439 (1981) 30-40;

E.Date, M.Jimbo, M.Kashiwara, T.Miwa, Transformation groups for soliton equations, in: Proc.RIMS symp. Nonlinear integrable systems - classical theory and quantum theory, M.Jimbo, T.Miws, eds., World Scientific, Singapore 1983, p.39;

M. Jimbo, T. Miwa, Publ.RIMS, Kyoto Univ,. 19 (1983) 943-1001 
[3] J.Wess and B.Zumino, Phys.Lett. B37 (1971) 95;

E.Witten, Nucl.Phys. B223 (1983) 422421; Comm.Math.Phys. 92 (1984) 455472;

S.P.Novikov, Sov.Math.Dokl. 24 (1981) 222226; Russian Mathematical Surveys 37 (1982) 19

[4] A.Gerasimov, A.Marshakov, A.Morozov, M.Olshanetsky, S. Shatashvili, Int.J.Mod.Phys. A5 (1990) 24952589

[5] A.Gerasimov, S.Khoroshkin, D.Lebedev, A.Mironov and A.Morozov, Int.J.Mod.Phys. A10 (1995) 25892614, hep-th/9405011;

S.Kharchev, A.Mironov and A.Morozov, Theor.Math.Phys. 104 (1995) 129-143, q-alg/9501013;

A.Mironov, hep-th/9409190; Theor.Math.Phys. 114 (1998) 127, q-alg/9711006

[6] S.-S.Chern and J.Simons, Ann.Math. 99 (1974) 48-69

[7] E.Witten, Comm.Math.Phys. 121 (1989) 351

[8] E.Guadagnini, M.Martellini and M.Mintchev, Clausthal 1989, Procs.307-317; Phys.Lett. B235 (1990) 275; N.Yu.Reshetikhin and V.G.Turaev, Comm. Math. Phys. 127 (1990) 1-26

[9] P.Ramadevi, T.R.Govindarajan and R.K.Kaul, Nucl.Phys. B402 (1993) 548-566, hep-th/9212110; Nucl.Phys. B422 (1994) 291-306, hep-th/9312215

[10] A.Morozov and A.Smirnov, Nucl.Phys. B835 (2010) 284-313, arXiv:1001.2003

[11] A.Mironov, A.Morozov and An.Morozov, Strings, Gauge Fields, and the Geometry Behind: The Legacy of Maximilian Kreuzer, World Scietific Publishins Co.Pte.Ltd. 2013, pp.101-118, arXiv:1112.5754; JHEP 03 (2012) 034, arXiv:1112.2654

[12] M.Atiyah, The geometry and physics of knots, (CUP, 1990)

[13] L.Alday, D.Gaiotto and Y.Tachikawa, Lett.Math.Phys. 91 (2010) 167-197, arXiv:0906.3219

[14] N.Wyllard, JHEP 0911 (2009) 002, arXiv:0907.2189

[15] A.Mironov and A.Morozov, Nucl.Phys. B825 (2009) 1-37, arXiv:0908.2569

[16] R.Dijkgraaf and C.Vafa, arXiv:0909.2453;

T.Eguchi and K.Maruyoshi, arXiv:0911.4797; arXiv:1006.0828;

H.Itoyama, K.Maruyoshi and T.Oota, Prog.Theor.Phys. 123 (2010) 957-987, arXiv:0911.4244

[17] A.Mironov, A.Morozov and Sh.Shakirov, JHEP 02 (2010) 030, arXiv:0911.5721; Int.J.Mod.Phys. A25 (2010) 3173-3207, arXiv:1001.0563; JHEP 1103 (2011) 102, arXiv:1011.3481;

A.Mironov, Al.Morozov and And.Morozov, Nucl.Phys. B843 (2011) 534-557, arXiv:1003.5752

[18] A.Mironov, A.Morozov and Sh.Shakirov, JHEP 1103 (2011) 102, arXiv:1011.3481; JHEP 1102 (2011) 067, arXiv:1012.3137;

A.Morozov and A.Smironov, arXiv:1012.1312

[19] V.A.Alba, V.A.Fateev, A.V.Litvinov and G.M.Tarnopolsky, Lett.Math.Phys. 98 (2011) 33-64, arXiv:1012.1312;

A.Belavin and V.Belavin, Nucl.Phys. B850 (2011) 199-213, arXiv:1102.0343;

S.Mironov, An.Morozov and Y.Zenkevich, JETP Lett. 99 (2014) 115, arXiv:1312.5732;

Y.Matsuo, C.Rim and H.Zhang, arXiv:1405.3141

[20] H.Awata and Y.Yamada, JHEP 1001 (2010) 125, arXiv:0910.4431; arXiv:1004.5122;

S.Yanagida, arXiv:1005.0216

[21] A.Mironov, A.Morozov, Sh.Shakirov and A.Smirnov, Nucl.Phys. B855 (2012) 128-151, arXiv:1105.0948

[22] H.Itoyama, T.Oota and R.Yoshioka, arXiv:1308.2068

[23] G.Moore, N.Nekrasov, S.Shatashvili, Nucl.Phys. B534 (1998) 549-611, hep-th/9711108; hep-th/9801061; A.Losev, N.Nekrasov and S.Shatashvili, Commun.Math.Phys. 209 (2000) 97-121, hep-th/9712241; ibid. 77-95, hep-th/9803265 
[24] N.Nekrasov, Adv.Theor.Math.Phys. 7 (2004) 831-864;

R.Flume and R.Pogossian, Int.J.Mod.Phys. A18 (2003) 2541;

N.Nekrasov and A.Okounkov, hep-th/0306238

[25] V.Pestun, arXiv:0906.0638

[26] A.Mironov and A.Morozov, Phys.Lett. B680 (2009) 188-194, arXiv:0908.2190

[27] M.Bershtein and O.Foda, arXiv:1404.7075;

K.Alkalaev and V.Belavin, arXiv:1404.7094

[28] Al.B. Zamolodchikov, Sov.Phys. JETP 63(5) (1986) 1061-1066; Theor.Math.Phys. 73 (1987) 1088

[29] A.Marshakov, A.Mironov and A.Morozov, JHEP 11 (2009) 048, arXiv:0909.3338

[30] R.Poghossian, JHEP 0912 (2009) 038, arXiv:0909.3412

[31] Al.Zamolodchikov, Comm.Math.Phys. 96 (1984) 419

[32] Al.Zamolodchikov, Nucl.Phys. B285 (1987) 481

[33] G.Belyi, Mathematics of the USSR: Izvestiya, 14:2 (1980) 247-256

A.Grothendieck, Sketch of a Programme, Lond. Math. Soc. Lect. Note Ser. 242 (1997) 243-283; Esquisse d'un Programme, in: P.Lochak, L.Schneps (eds.), Geometric Galois Action, pp.5-48, Cambridge University Press, Cambridge (1997)

[34] D.Friedan and S.Shenker, Phys.Lett. 175B (1986) 287;

N.Ishibashi, Y.Matsuo amd H.Ooguri, Mod.Phys.Lett. 2A (1987) 119;

L.Alvarez-Gaume, C.Gomez and C.Reina, Phys.Lett. 190B (1987) 55;

E.Witten, Comm.Math.Phys. 113 (1988) 529;

A.Morozov, Phys.Lett. 196B (1987) 325

[35] A.Levin and A.Morozov, Phys.Lett. B243 (1990) 207-214

[36] A.Mironov, A.Morozov and S.Natanzon, Theor.Math.Phys. 166 (2011) 1-22, arXiv:0904.4227; Journal of Geometry and Physics 62 (2012) 148-155, arXiv:1012.0433

[37] S.Kharchev, A.Marshakov, A.Mironov and A.Morozov, Int.J.Mod.Phys. A10 (1995) 2015, hep-th/9312210; A.Orlov and D.M.Shcherbin, Theor.Math.Phys. 128 (2001) 906-926;

A.Orlov, Theor.Math.Phys. 146 (2006) 183206;

A.Alexandrov, A.Mironov, A.Morozov and S.Natanzon, J.Phys. A: Math.Theor. 45 (2012) 045209, arXiv:1103.4100

[38] A.Alexandrov, A.Mironov, A.Morozov and S.Natanzon, arXiv:1405.1395

[39] N.Nemkov, to appear

[40] Al.Zamolodchikov, private communication

[41] B.Ponsot and J.Teschner, arXiv:hep-th/9911110;

B.Ponsot and J.Teschner, Commun.Math.Phys. 224 (2001) 613-655, arXiv:math/0007097

[42] V.Knizhnik and A.Morozov, JETP Lett., 39 (1984) 240;

H.Levine and S.Libby, Phys.Lett., B150 (1985) 182

[43] N.Seiberg and E.Witten, Nucl.Phys., B426 (1994) 19-52, hep-th/9408099; Nucl.Phys., B431 (1994) 484550 , hep-th/9407087

[44] A.Gorsky, I.Krichever, A.Marshakov, A.Mironov, A.Morozov, Phys.Lett., B355 (1995) 466-477, hepth/9505035;

R.Donagi and E.Witten, Nucl.Phys., B460 (1996) 299-334, hep-th/9510101

[45] H.Itoyama and A.Morozov, Nucl.Phys., B477 (1996) 855-877, hep-th/9511126; Nucl.Phys., B491 (1997) 529-573, hep-th/9512161, hep-th/9601168;

A.Gorsky and A.Mironov, hep-th/0011197 
[46] Vl.Dotsenko and V.Fateev, Nucl.Phys., B240 (1984) 312-348

[47] B.Feigin and D.Fuks, Funct.Anal.Appl. 16 (1982) 114-126 (Funkt.Anal.Pril. 16 (1982) 47-63)

[48] M.Wakimoto, Commun.Math.Phys. 104 (1986) 605-609

[49] P.Zograf, arXiv:1312.2538

[50] R. Hirota, Phys.Rev.Lett. 27 (1971) 1192;

Y.Ohta, J.Satsuma, D.Takahashi and T.Tokihiro, Prog. Theor. Phys. Suppl. 94 (1988) 210

[51] G.Moore and N.Seiberg, Phys.Lett. B220 (1989) 422

[52] A.Marshakov, A.Mironov and A.Morozov, Theor.Math.Phys. 164 (2010) 831-852 (Teor.Mat.Fiz.164:327,2010), arXiv:0907.3946

[53] For a detailed review see:

Andrey Mironov, Sergey Mironov, Alexei Morozov and Andrey Morozov, Theor.Math.Phys. 165 (2010) 1662-1698 (Teor.Mat.Fiz. 165 (2010) 503-542), arXiv:0908.2064

[54] K.W.J.Kadell, Compositio Math. 87 (1993) 5-43; Adv.Math. 130 (1997) 33-102;

J.Kaneko, SIAM.J.Math.Anal. 24 (1993) 1086-1110

[55] H.Itoyama and T.Oota, arXiv:1003.2929

[56] S.Kharchev, A.Marshakov, A.Mironov, A.Morozov and S.Pakuliak, Nucl.Phys. B404 (1993) 717-750, hepth/9208044;

A.Mironov and S.Pakuliak, Int.J.Mod.Phys. A8 (1993) 3107-3137, hep-th/9209100;

H.Awata, Y.Matsuo, S.Odake and J.Shiraishi, Soryushiron Kenkyu 91 (1995) A69-A75, hep-th/9503028

[57] D.Galakhov, A.Mironov and A.Morozov, arXiv:1311.7069, to appear in JHEP

[58] D.Galakhov, A.Mironov and A.Morozov, JHEP 2012 (2012) 67, arXiv:1205.4998

N.Nemkov, J. Phys. A: Math. Theor. 47 (2014) 105401, arXiv:1307.0773;

M.Billo, M.Frau, L.Gallot, A.Lerda and I.Pesando, arXiv:1302.0686; arXiv:1307.6648

[59] N.Iorgov, O.Lisovyy and Yu.Tykhyy, arXiv:1308.4092

[60] I.S.Gradshteyn,I.M.Ryzhik, Tables of Integrals, Series and Products, Academic Press, 1980

[61] A.Marshakov, A.Mironov and A.Morozov, J.Geom.Phys. 61 (2011) 1203-1222, arXiv:1011.4491

[62] A.Alexandrov, A.Mironov and A.Morozov, Int.J.Mod.Phys. A21 (2006) 2481-2518, hep-th/0412099; Fortsch.Phys. 53 (2005) 512-521, hep-th/0412205 